\title{
A survey of the ISM in early-type galaxies ${ }^{\star}$. I. The ionized gas
}

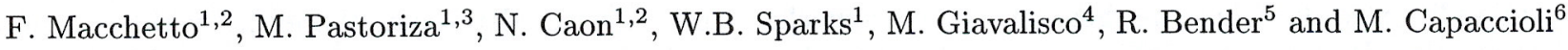 \\ 1 Space Telescope Science Institute, Baltimore MD, U.S.A. \\ 2 Affiliated to the Astrophysics Division, Space Science Department, ESA \\ 3 Instituto de Fisica, UFRGS, Porto Alegre, Brazil \\ 4 The Observatory of the Carnegie Institution of Washington, Pasadena, CA, U.S.A. - Hubble Fellow \\ 5 Universitäts-Sternwarte, München, Germany \\ 6 Osservatorio Astronomico di Capodimonte, Napoli, Italy
}

Received December 1, 1995; accepted May 2, 1996

\begin{abstract}
We present results of a CCD optical imaging survey of the ionized gas in 73 luminous elliptical and lenticular galaxies, selected from the RC3 catalog to represent a broad variety of X-ray, radio, infrared and kinematical properties. For each galaxy we have used broad-band $\mathrm{R}$ images and narrow-band images centered at the H $\alpha$ and [NII] emission lines to derive the luminosity and distribution of the ionized gas. We found that a large fraction of $E$ $(72 \%)$ and S0 (85\%) galaxies in our sample contain ionized gas. The gas morphology appears to be rather smooth for most galaxies; however $\sim 12 \%$ of the sample galaxies show a very extended filamentary structure. According to the morphology and size of the gas distribution, the galaxies have been classified into three broad groups, named small disk (SD), regular extended $(\mathrm{RE})$ and filamentary structure $(\mathrm{F})$. The mean diameter of the emitting region ranges between 1 and $10 \mathrm{kpc}$; the derived mass of the ionized gas ranges between $10^{3}$ and $10^{5}$ solar masses. A significant correlation between $\mathrm{H} \alpha+[\mathrm{NII}]$ and X-ray luminosities is found for those galaxies (27\% of the sample) for which we have detected ionized gas and are also listed as X-ray sources. However, there are relatively strong X-ray emitting galaxies for which we have not detected $\mathrm{H} \alpha+[\mathrm{NII}]$ emission and objects which show emission-lines but are not listed either in the EINSTEIN or in the ROSAT databases. The distribution of datapoint and upper limits in this diagram suggests that galaxies with warm gas are also X-ray emitters, while there are X-ray emitters without measurable $\mathrm{H} \alpha+[\mathrm{NII}]$ emission. Similar characteristics are present in the correlation between the infrared luminosity in the $12 \mu \mathrm{m}$ band and $L_{\mathrm{H} \alpha+[\mathrm{NII}]}$; correlations with other infrared wavelengths are weaker. A strong correlation was also found between the $\mathrm{H} \alpha+[\mathrm{NII}]$ luminosity and the luminosity in the $B$ band inside the region occupied by the lineemitting gas. We use these correlations to discuss the possible mechanisms responsible for the gas ionization and excitation, analyzing in particular the role of the post-AGB stars and the thermal conduction from the X-ray halo in providing the necessary source of ionization.
\end{abstract}

Key words: galaxies: elliptical and lenticular - galaxies: ISM - galaxies: structure

\section{Introduction}

Optical surveys have shown that a large percentage of early-type galaxies contain extended dust lanes, patches or a central disk-like dust structure (Sadler \& Gerhardt 1985; Sparks et al. 1985; Macchetto \& Sparks 1992; Goudfrooij et al. 1994, hereinafter GHJN). In addition to the dust, warm $\left(10^{4} \mathrm{~K}\right)$ ionized gas is present in a high fraction of ellipticals, of the order of $50 \%$, as shown by spectroscopic and/or imaging surveys. The amount of ionized gas is of

Send offprint requests to: F. Macchetto

${ }^{\star}$ Based on observations collected at the European Southern Observatory, within the ESO Key-Programme 1-004-43K the order of $10^{3}-10^{4}$ solar masses (Caldwell 1984; Phillips et al. 1986, hereinafter PJDSB; GHJN). Although relatively small in mass, the contribution to the energy budget of the galaxy can be very large. The optical line-emission can dominate, at least locally, the energy loss from Xrays (Sparks et al. 1989, hereinafter SMG) and therefore it has to be understood in order to derive a correct description for the energetics of the interstellar medium in these galaxies.

The nature, the origin and the future evolution of the gas and dust in elliptical galaxies is still a highly controversial subject. A first scenario is one in which the ISM is a normal, quiescent component of these early-type 
galaxies. An alternative scenario is that in which clouds of cold gas and dust have been accreted in merger events and heated by thermal conduction from the X-ray emitting plasma (SMG; Kim 1989). Finally there is the coolingflow scenario in which the stellar mass-loss, heated at high temperatures $\left(10^{6}-10^{7} \mathrm{~K}\right)$ by supernovae explosions during the galaxy formation, cools and condenses into filaments (see Fabian 1994, for a review).

In addition to the origin, neither the excitation mechanism nor the ultimate fate of this gas are well established. Photoionization by hot young stars (Kim 1989; Shields 1991, hereinafter S91), by hot old stars (di Serego Alighieri et al. 1990; Binette et al. 1994, hereinafter BMSB) or radiation originating in an active nucleus (Fosbury et al. 1982) are among the competing theories, along with shocks (Heckman et al. 1989). Ionization linked with X-ray emitting gas is another alternative proposed to explain the observed emission lines and their excitation character (SMG; Donahue \& Voit 1991).

In order to study the properties of the interstellar medium in early-type galaxies and elucidate its origin, we have carried out an extensive program of imaging and spectroscopic observations of a large sample of bright elliptical and lenticular galaxies. The galaxies in our sample have been selected so as to span a wide range of optical, Xray, radio, infrared and kinematical properties. The observations have been aimed at reaching fainter limiting-flux levels than in previous studies, in order to provide a more complete and extensive mapping of the ionized-gas distribution, especially of those faint filamentary structures extending far out from the galaxy nucleus.

In this paper we present results of CCD observations for 39 ellipticals and 34 lenticular galaxies. For each galaxy we have obtained the total luminosity, morphology and extension of the ionized gas. We have explored the correlations between the ionized gas luminosity and other global properties of the galaxies, e.g. X-ray, IR and radio emission, available in the literature and electronic databases. Although a thorough and complete statistical analysis of these correlations is outside the scope of the present paper, the results shown here provide valuable information on the physics of the ionized gas. In particular, we discuss the viability of UV photons emitted by post-AGB stars and the conductive heating by hot electrons associated with an X-ray emitting coronal halo as the dominant ionizing sources.

This paper is structured as follows: in Sect. 2 we present the galaxy sample, in Sect. 3 we describe the observations and data reduction. The morphology of the ionized gas is presented in Sect. 4. In Sect. 5 we analyze the correlations between the $\mathrm{H} \alpha+[\mathrm{NII}]$ luminosities and X-ray, IR and radio fluxes. The possible mechanisms for the ionization and excitation of the gas are briefly discussed in Sect. 6. Concluding remarks are given in Sect. 7.

\section{The sample}

The early-type galaxies studied in this paper are all luminous galaxies $\left(B_{\mathrm{T}} \lesssim 13.0 \mathrm{mag}\right)$, with morphological type E or S0 (including S0/Sa) according to both the Third Reference Catalogue of Bright Galaxies (RC3; de Vaucouleurs et al. 1991) and the Revised Shapley-Ames Catalog of Bright Galaxies (RSA; Sandage \& Tammann 1987), selected to span the entire variety of morphological types and isophotal shapes from E0 through S0/Sa, to provide a fair interval of optical luminosity, and to include both radio-loud and radio-quiet, X-ray emitters and non-emitters.

The galaxies in our sample are listed in Table 1, along with their morphological types, magnitudes, and extinction in the blue band taken from the RC3 catalogue. The distances have been derived from the " 220 model" for the Virgo infall of Kraan-Korteweg (1986), assuming a Virgo Cluster distance of $21.3 \mathrm{Mpc}$, which corresponds to a Hubble constant $H_{0}=55 \mathrm{~km} \mathrm{~s}^{-1} \mathrm{Mpc}^{-1}$ (see Jerjen \& Tammann 1993). For galaxies belonging to a group or cluster, the same distance has been taken, following Davies et al. (1987). For those galaxies not present in KraanKorteweg's compilation, the distance has been derived from the redshift reduced to the reference frame defined by the cosmic microwave background (from RC3).

The luminosity distribution for the galaxy sample is presented in Fig. 1: the absolute blue magnitudes of our galaxies range between -19.0 and -23.5 , with a maximum at $M_{B}=-21.5$.

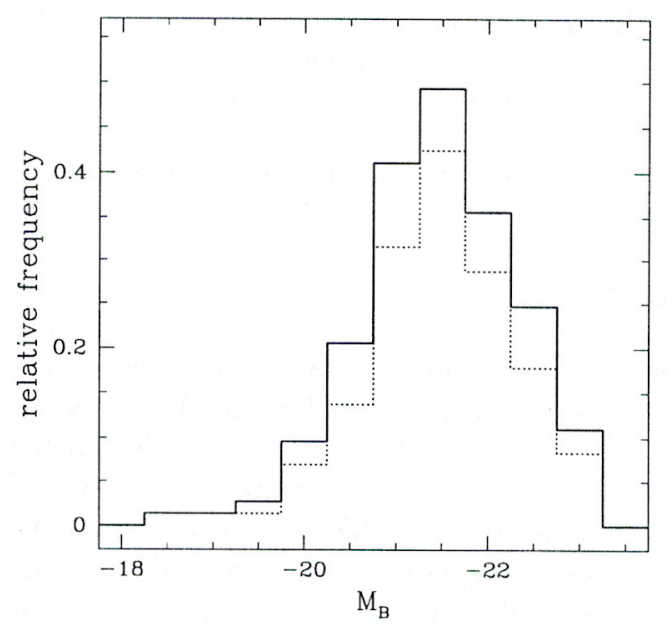

Fig. 1. The luminosity function of our galaxy sample. The dashed histogram refers to those galaxies with positive $\mathrm{H} \alpha+[\mathrm{NII}]$ detection 
Table 1. The galaxy sample

\begin{tabular}{|c|c|c|c|c|c|}
\hline Ident & $\mathrm{RA}$ & $(2000)$ & type & $\overline{B_{\mathrm{T}}^{0}}$ & $A_{B}$ \\
\hline NGC 524 & 012447.8 & +093221 & $\mathrm{~S} 0 / \mathrm{Sa}$ & 11.17 & 0.03 \\
\hline NGC 533 & 012531.5 & +014535 & E3 & 12.22 & 0.06 \\
\hline NGC 584 & 013120.9 & -065206 & So & 11.21 & 0.14 \\
\hline NGC 596 & 013252.1 & -070157 & E0/S0 & 11.66 & 0.11 \\
\hline NGC 636 & 013906.6 & -073047 & E1 & 12.22 & 0.13 \\
\hline NGC 720 & 015300.4 & -134421 & E5 & 11.13 & 0.00 \\
\hline NGC 741 & 015620.9 & +053744 & E0 & 12.07 & 0.14 \\
\hline NGC 821 & 020821.1 & +105944 & E6 & 11.72 & 0.17 \\
\hline NGC 1172 & 030136.1 & -145013 & So & 12.56 & 0.11 \\
\hline NGC 1399 & 033829.0 & -352658 & E1 & 10.44 & 0.00 \\
\hline NGC 1400 & 033931.3 & -184119 & S0 & 11.89 & 0.14 \\
\hline NGC 1407 & 034012.4 & -183452 & E0/S0 & 10.71 & 0.17 \\
\hline NGC 1453 & 034627.3 & -035810 & E0 & 12.26 & 0.25 \\
\hline NGC 1600 & 043139.9 & -050516 & E4 & 11.83 & 0.08 \\
\hline NGC 2865 & 092330.8 & -230948 & $\mathrm{E} 4$ & 12.18 & 0.29 \\
\hline NGC 3115 & $\begin{array}{lll}10 & 05 & 14.1\end{array}$ & -074307 & S0 & 9.74 & 0.10 \\
\hline NGC 3268 & 103000.6 & -351930 & $\mathrm{E} 2$ & 12.26 & 0.28 \\
\hline NGC 3311 & 103643.3 & -273141 & So & 12.22 & 0.18 \\
\hline NGC 3379 & 104749.9 & +123457 & E0 & 10.18 & 0.05 \\
\hline NGC 3489 & 110018.2 & +135408 & $\mathrm{~S} 0 / \mathrm{Sa}$ & 11.15 & 0.02 \\
\hline NGC 3607 & 111654.1 & +180312 & So & 10.79 & 0.00 \\
\hline NGC 4105 & $\begin{array}{lll}12 & 06 & 40.7\end{array}$ & -294542 & So & 11.34 & 0.25 \\
\hline NGC 4472 & 122946.5 & +075958 & E1/S0 & 9.33 & 0.00 \\
\hline NGC 4473 & $12 \quad 2948.8$ & +132549 & E5 & 11.03 & 0.07 \\
\hline NGC 4486 & 123049.7 & +122324 & E0 & 9.49 & 0.10 \\
\hline NGC 4552 & 123539.9 & +123325 & S0 & 10.57 & 0.15 \\
\hline NGC 4636 & 124249.8 & +024117 & E0/S0 & 10.43 & 0.05 \\
\hline NGC 4782 & 125435.8 & -123406 & $\mathrm{EO}$ & 12.56 & 0.07 \\
\hline NGC 4936 & 130416.4 & -303129 & $\mathrm{E} 2$ & 11.36 & 0.36 \\
\hline NGC 5044 & 131524.0 & -162309 & E0 & 11.67 & 0.12 \\
\hline NGC 5077 & 131931.7 & -123926 & So & 12.21 & 0.08 \\
\hline NGC 5090 & 132113.5 & -434218 & $\mathrm{E} 2$ & 11.97 & 0.52 \\
\hline NGC 5102 & 132157.8 & -363747 & S0 & 9.99 & 0.22 \\
\hline NGC 5419 & 140338.6 & -335841 & SO & 11.44 & 0.25 \\
\hline NGC 5532 & 141652.8 & +104824 & So & 12.77 & 0.00 \\
\hline NGC 5812 & 150055.8 & -072729 & E0 & 11.83 & 0.32 \\
\hline NGC 5813 & 150111.2 & +014208 & E1 & 11.42 & 0.16 \\
\hline NGC 5831 & 150407.2 & +011315 & $\mathrm{E} 4$ & 12.31 & 0.15 \\
\hline NGC 5846 & 150629.4 & +013625 & So & 10.91 & 0.15 \\
\hline NGC 5864 & 150933.8 & +030311 & SB0 & 12.63 & 0.11 \\
\hline NGC 5898 & $\begin{array}{lll}15 & 18 & 13.3\end{array}$ & -240549 & S0 & 11.92 & 0.51 \\
\hline NGC 5903 & 151836.3 & -240406 & E3/S0 & 11.74 & 0.51 \\
\hline
\end{tabular}

\section{Observations and data reduction}

\subsection{Observations}

The observations were carried out during several runs at the European Southern Observatory (La Silla, Chile) with the $3.6 \mathrm{~m}$ and the NTT telescopes. CCD images were acquired both through a broad-band $R$ filter matching the Cousins standard band and through a narrow-band filter centered on the $\mathrm{H} \alpha+[\mathrm{NII}]$ emission lines. The instruments and setups used in the various observing runs are shown in Table 2; the filters' specifications are shown in Table 3. The exposure times were selected in order to achieve good signal-to-noise ratios in each of the two bands and to reach faint surface brightness levels. Two or more frames with each filter were taken for each galaxy to allow for easy removal of cosmic ray events. Typically, $\mathrm{H} \alpha$ frames were exposed 25-45 min, while $R$ images have exposure times in the range $30 \mathrm{~s}-3 \mathrm{~min}$. The images have been processed with the Image Reduction and Analysis Facility (IRAF), using the standard techniques for bias and darkcurrent subtraction, division by normalized twilight flatfields, and interpolation over bad columns and/or pixels. The sky level was determined by calculating the average intensity in blank regions in the frames, and subsequently subtracted out. While this value may be somewhat affected by contamination by the light from the galaxy, the residual in the sky background subtraction cancel out in the subtraction of the rescaled continuum image from the narrow-band image (see below). The residual cosmic rays 
Table 1. continued

\begin{tabular}{|c|c|c|c|c|c|}
\hline Ident & RA & $(2000)$ & type & $B_{\mathrm{T}}^{0}$ & $A_{B}$ \\
\hline$\overline{\mathrm{NGC}} 5920$ & 152151.8 & +074232 & S0 & 14.37 & 0.08 \\
\hline NGC 6034 & $\begin{array}{lll}16 & 03 & 32.4\end{array}$ & +171153 & $\mathrm{E} ?$ & 14.38 & 0.05 \\
\hline NGC 6305 & 171800.5 & -591018 & $\mathrm{E} / \mathrm{SO}$ & 12.58 & 0.54 \\
\hline NGC 6407 & 171800.5 & -591018 & $\mathrm{E}$ & 12.43 & 0.37 \\
\hline NGC 6483 & 175929.8 & -634007 & $\mathrm{E}$ & 12.63 & 0.33 \\
\hline NGC 6721 & 190050.5 & -574528 & E1 & 12.68 & 0.28 \\
\hline NGC 6758 & $1913 \quad 52.3$ & -561833 & $\mathrm{E} 2$ & 12.31 & 0.22 \\
\hline NGC 6776 & 192519.4 & -635141 & E1 pec & 12.71 & 0.20 \\
\hline NGC 6851 & 200333.6 & -481702 & $\mathrm{E} 4$ & 12.51 & 0.17 \\
\hline NGC 6861 & $2007 \quad 19.2$ & $\begin{array}{lll}-48 & 22 & 12\end{array}$ & S0 & 11.92 & 0.15 \\
\hline NGC 6868 & $\begin{array}{lll}20 & 09 & 53.8\end{array}$ & -482245 & $\mathrm{E} 3 / \mathrm{S} 0$ & 11.49 & 0.15 \\
\hline NGC 6875 & $2013 \quad 12.4$ & -460938 & So & 12.78 & 0.00 \\
\hline NGC 6876 & $\begin{array}{llll}20 & 18 & 20.3\end{array}$ & -705128 & E3 & 11.83 & 0.17 \\
\hline NGC 6909 & $\begin{array}{llll}20 & 27 & 38.7\end{array}$ & -470134 & E5 & 12.51 & 0.07 \\
\hline NGC 6936 & 203556.6 & -251646 & SA0 & 13.52 & 0.19 \\
\hline NGC 6964 & 204724.0 & +001802 & E pec & 13.38 & 0.39 \\
\hline NGC 7014 & 210752.6 & -471040 & E5 & 13.02 & 0.07 \\
\hline NGC 7029 & 211152.4 & -491658 & S0 & 12.42 & 0.00 \\
\hline NGC 7041 & 211632.6 & -482151 & $\mathrm{~S} 0 / \mathrm{E} 7$ & 12.19 & 0.07 \\
\hline NGC 7049 & 211900.3 & -483350 & $\mathrm{~S} 0 / \mathrm{Sa}$ & 11.57 & 0.03 \\
\hline NGC 7192 & 220650.0 & -641857 & So & 12.23 & 0.00 \\
\hline NGC 7196 & $2205 \quad 54.7$ & -500711 & E3/S0 & 12.35 & 0.00 \\
\hline NGC 7562 & $23 \quad 15 \quad 57.4$ & +064115 & E2 & 12.37 & 0.23 \\
\hline NGC 7619 & 232014.7 & +081223 & E3 & 11.93 & 0.17 \\
\hline NGC 7626 & 232042.4 & +08 1302 & E1 & 12.06 & 0.17 \\
\hline NGC 7796 & 235859.8 & -552724 & E1 & 12.39 & 0.00 \\
\hline IC 1459 & 225709.5 & -362737 & $\mathrm{E}$ & 10.83 & 0.04 \\
\hline IC 4296 & 133638.9 & -335759 & E0 & 11.42 & 0.13 \\
\hline IC 4797 & $\begin{array}{lll}18 & 56 & 29.3\end{array}$ & -541822 & E pec & 11.85 & 0.31 \\
\hline IC 4889 & 194515.9 & -542037 & S0 & 11.91 & 0.19 \\
\hline IC 5105 & 212422.2 & -403211 & E5 & 12.42 & 0.07 \\
\hline
\end{tabular}

Notes to the table:

Coordinates, blue apparent magnitudes $B_{\mathrm{T}}^{0}$, corrected for galactic and internal absorption and for redshift, and galactic absorption in the $B$-band $A_{B}$ have been taken from RC3.

The morphological type is as listed in the RSA catalog.

present in the frames were identified as those pixels whose values are larger than the local mean value by more than 5 sigma, and replaced by a median value calculated over the nearest $5 \times 5$ pixels.

\subsection{Emission-line images generation}

Several data reduction steps have to be performed before generating emission-line intensity images. The first step is to align, for each galaxy, the broad-band and narrowband images to an accuracy of a few hundredths of a pixel. This was achieved by measuring the centers of the common stars in the images, after fitting gaussians to the stars profiles, and then registering and convolving the image(s) with the best seeing to match the seeing of the other image. This minimizes the degradation of the resolution in our data. For each galaxy, a final emission-line and a final $R$ image were thus produced, summing the images in each set. In turn, these two final images were aligned and brought to the same resolution.
The most important step in the data reduction process, as well as the principal source of error, is the determination of the intensity scale factor that has to be applied to the final $R$ image in order to match the stellar continuum in the final narrow-band image, so as to give the pure emission $\mathrm{H} \alpha+[\mathrm{NII}]$ image once it is subtracted out.

The final $R$ scaled image was obtained through the following procedure. Ellipses were fitted to the isophotes for both the combined $R$ and emission-line images using the task "ellipse" of the Space Telescope Science Data Analysis System (STSDAS), which produces a table containing the isophotal parameters. These data allow us to plot, for the isophotes with the same major semidiameter $a$, the corresponding mean count levels $N_{R}$ versus $N_{\mathrm{NB}}$. A least square fit was made to the outer isophotes, in the radial range where the stellar continuum light dominates, avoiding the inner regions where the signal from the ionized gas is expected to be significant. The slope of the fit gives the scale factor $\varepsilon$ between the continuum flux in the $R$ band and that in the emission-line band, while the 
Table 2. Observational set-ups

\begin{tabular}{|c|c|c|c|c|c|}
\hline \multirow{2}{*}{$\begin{array}{l}\text { Run } \\
\text { Oct } 3-6,1989\end{array}$} & \multicolumn{2}{|c|}{$\begin{array}{l}\text { Instrument \& detector } \\
\text { pixel size \& FOV }\end{array}$} & \multicolumn{2}{|c|}{ ESO filters } & \multirow{2}{*}{ 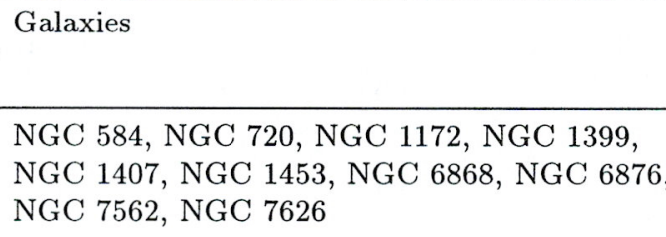 } \\
\hline & $\begin{array}{l}3.6 \mathrm{~m}+\mathrm{EFOSC} \\
0.337\end{array}$ & $\begin{array}{r}\mathrm{CCD \# 8} \\
3^{\prime} .7 \times 5^{\prime} .8\end{array}$ & 388,389 & 554 & \\
\hline Mar 30-Apr 11990 & $\begin{array}{l}\text { NTT+EFOSC2 } \\
0 . ' 257\end{array}$ & $\begin{array}{r}\mathrm{CCD \# 5} \\
1^{\prime} .4 \times 2^{\prime} .2\end{array}$ & $\begin{array}{l}388,436 \\
438,439 \\
441\end{array}$ & 585 & 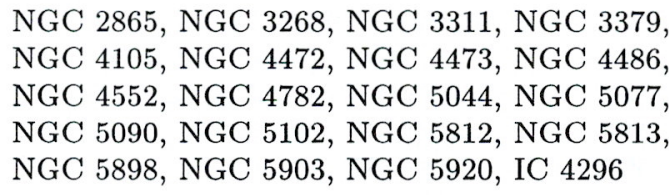 \\
\hline Aug $17-21,1990$ & $\begin{array}{l}\text { NTT+EFOSC2 } \\
0 . \prime 300 \text { (binned) }\end{array}$ & $\begin{array}{l}\mathrm{CCD} \# 17 \\
2^{\prime} .7 \times 2^{\prime} .6\end{array}$ & $\begin{array}{l}388,438 \\
439\end{array}$ & 585 & $\begin{array}{l}\text { NGC } 524 \text {, NGC 596, NGC 636, NGC } 741 \text {, } \\
\text { NGC } 821 \text {, NGC } 1400 \text {, NGC 5846, NGC } 6034 \text {, } \\
\text { NGC } 6305 \text {, NGC 6407, NGC 6483, NGC 6758, } \\
\text { NGC } 6875 \text {, NGC 6909, NGC 7014, NGC 7041, } \\
\text { NGC } 7049 \text {, NGC 7192, NGC 7619, NGC 7796, } \\
\text { IC 4797, IC 4889, IC 5105 }\end{array}$ \\
\hline May $12-16,1991$ & $\begin{array}{l}\text { NTT+EMMI } \\
0^{\prime \prime} 350\end{array}$ & $\begin{array}{r}\mathrm{CCD} \# 24 \\
9^{\prime} .2 \times 8^{\prime} \cdot 6\end{array}$ & $\begin{array}{l}595,596 \\
597,598\end{array}$ & 608 & $\begin{array}{l}\text { NGC } 3115 \text {, NGC } 3489 \text {, NGC } 3607 \text {, NGC } 4636 \text {, } \\
\text { NGC } 4936 \text {, NGC 5419, NGC 5532, NGC } 6776 \text {, } \\
\text { NGC } 7029\end{array}$ \\
\hline Aug 21-24, 1993 & $\begin{array}{l}\text { NTT+EMMI } \\
0 .{ }^{\prime \prime} 340\end{array}$ & $\begin{array}{l}\mathrm{CCD} \# 34 \\
9 ! 2 \times 8^{\prime} \cdot 6\end{array}$ & $\begin{array}{l}595,597 \\
598,653\end{array}$ & 608 & $\begin{array}{l}\text { NGC } 533 \text {, NGC } 1600 \text {, NGC } 5831 \text {, NGC } 5864 \text {, } \\
\text { NGC } 6721 \text {, NGC } 6851 \text {, NGC } 6861 \text {, NGC } 6936 \text {, } \\
\text { NGC } 6964 \text {, NGC } 7196 \text {, IC } 1459\end{array}$ \\
\hline
\end{tabular}

intercept $k$, usually close to 0 , depends on the residuals in the sky background in the two images.

$$
N_{R}=\varepsilon \times N_{\mathrm{NB}}+k
$$

In some cases, a visual inspection of the continuumsubtracted image made it necessary to iterate the fit adjusting the radial range so as to completely exclude from the fit regions affected by line emission. The formal uncertainty in the scale factor is usually less than $1 \%$; for a few cases, especially for galaxies with extended emission, we found uncertainties as large as $2-3 \%$.

There were a few cases, for instance IC 4889 and NGC 5044, where the scale factor could not be accurately determined using the method described above, because the region of the galaxy devoid of line emission was too small to give reliable results. We determined the scale factor $\varepsilon$ by measuring the mean values inside boxes of $10 \times 10$ pixels whose location was carefully chosen to avoid the presence of ionized gas, and then performing a least square fit between the mean values in the $R$ and $\mathrm{H} \alpha+[\mathrm{NII}] \mathrm{im}-$ ages.

The accuracy of the scale factor was checked, for all galaxies, on the continuum subtracted image, where the resulting values should be zero in the outer regions where there is no presence of ionized-gas emission.
In order to compute the total counts $N(\mathrm{H} \alpha+[\mathrm{NII}])$ for the $\mathrm{H} \alpha+[\mathrm{NII}]$ emission, we set to zero, in the pure emission-line images, all the pixel values under a given threshold (typically 1 to $2 \mathrm{rms}$ of the background level). This allows to better define the emitting region and to reduce the effect of possible inhomogeneities in the background when integrating the flux over a large area. This threshold was adjusted to the specific photometric quality of each image and to the characteristic of the emitting region (for instance, a low value was selected if required to avoid to clip-out the faint and diffuse emission seen in some of our objects).

We checked the consistency of this procedure by comparing the resulting $N(\mathrm{H} \alpha+[\mathrm{NII}])$ values obtained with different threshold levels. They change by less than $5 \%$ for thresholds varying from 0 (i.e. no threshold applied) to 2 sigma for those galaxies showing a concentrated, disk-like emission. However, for galaxies with extended and diffuse emission, this value can change by as much as $20-25 \%$, indicating that a high value for the threshold is not appropriate since it eliminates areas with true emission. In general, these threshold levels range from 0.1 to $1.010^{-16}$ $\mathrm{erg} \mathrm{s}^{-1} \mathrm{~cm}^{-2} \operatorname{arcsec}^{-2}$; individual threshold levels for each object are given in Figs. 9.

The relative error on the computed total $\mathrm{H} \alpha+[\mathrm{NII}] \mathrm{lu}-$ minosity associated with the uncertainty on $\varepsilon$ depends on 
Table 3. Filter specifications

\begin{tabular}{lccc}
\hline \hline $\begin{array}{l}\text { Filter number } \\
\text { ESO \# }\end{array}$ & $\begin{array}{c}\lambda_{\text {cent }} \\
(\AA)\end{array}$ & $\begin{array}{c}\text { FWHM } \\
(\AA)\end{array}$ & $\begin{array}{c}\text { Peak trans. } \\
(\%)\end{array}$ \\
\hline & & & \\
388 & 6597 & 77 & 86 \\
389 & 6646 & 80 & 84 \\
436 & 6560 & 63 & 70 \\
438 & 6632 & 69 & 67 \\
439 & 6686 & 82 & 58 \\
441 & 6832 & 74 & 66 \\
595 & 6587 & 73 & 54 \\
596 & 6547 & 73 & 53 \\
597 & 6620 & 66 & 63 \\
598 & 6665 & 66 & 59 \\
653 & 6579 & 32 & 55 \\
554 & 6438 & 1666 & 86 \\
585 & 6431 & 1654 & 87 \\
608 & 6390 & 1498 & 85 \\
\hline
\end{tabular}

the equivalent width of the ionized-gas emission, which may be varying across the gas distribution. Galaxies with faint and diffuse emission, having on average a small equivalent width, are those most affected, while galaxies with a strong and concentrated emission are the least sensitive. Further possible sources of error are the sky-subtraction, photon noise, residual spatial variations after flat-fielding, an inaccurate alignment and PSF matching between the two images, the selection of the threshold, the uncertainty on the zero-point calibration and the unknown line ratios (see below). Since an estimate of the total uncertainty on our final emission-line images that takes properly into account all of these potential source of errors would be quite impractical, we adopted a more heuristic and straightforward approach. We compared the results obtained for those few objects observed in two different observing runs (and thus with two different telescope, filter and detector combinations), or for the same set of images reduced independently by two different authors. The typical difference turned out to be of the order of $10-15 \%$, and occasionally somewhat larger for galaxies with faint extended emission. As we shall discuss later, comparison with previous published studies shows a much larger discrepancy.

We should note that, since the $\mathrm{H} \alpha$ and [NII] lines fall inside the $R$-passband, the subtraction of the rescaled $R$ image also implies a partial subtraction of the $\mathrm{H} \alpha+[\mathrm{NII}]$ emission. The magnitude of this effect is equal to the ratios of the effective widths of the narrow-band and $R$ filters used in the observations (we adopted an average value 0.04 for all filter combinations); the final counts have been corrected accordingly.
It also has to be noted that, because we match the seeing profile of the narrow-band and broad-band images to the lowest resolution achieved, we are insensitive to changes in the PSF. Furthermore, the matching technique and image normalization and calibration procedure make the final results insensitive to moderate changes in atmospheric transmission. The main effect of large changes in atmospheric transmission would be an increase in the value of the threshold and therefore a small reduction in the detection of the faintest emission features. The resolution of the final emission-line images is given in the captions to Figs. 9.

\subsection{Flux calibration}

The measured $\mathrm{H} \alpha+[\mathrm{NII}]$ counts have been converted into an absolute flux scale using the following procedure.

First we calibrated the continuum emission by measuring in our $R$ images a set of integrated counts inside centered circular apertures matching those listed in the photoelectric photometry catalogs (de Vaucouleurs \& Longo 1987; Poulain \& Nieto 1994). Using these data we determined the zero point $R_{0}$ by a least-square fit to the equation:

$$
R(<r)=R_{0}-2.5 \log N(<r)
$$

where $N(<r)$ is the number of counts inside a centered circular aperture of radius $r$, and $R(<r)$ is the corresponding magnitude.

The flux corresponding to a star of $R=0 \mathrm{mag}$ is $f_{0}=2.1310^{-9} \mathrm{erg} \mathrm{s}^{-1} \mathrm{~cm}^{-2} \AA^{-1}$ at $6500 \AA$ (in the Cousins photometric system). This factor has been computed with the task "calcphot" in IRAF/STSDAS, using the transmission curves of our $R$ filters and the spectral energy distribution of Vega. The conversion from counts to flux for the CCD $R$ image is thus given by:

$$
F_{R}=N_{R} 2.1310^{-9} 10^{-0.4 R_{0}} \quad \operatorname{erg~s}^{-1} \mathrm{~cm}^{-2} \AA^{-1}
$$

Replacing $N_{R}$ in this equation by (1) we can derive the flux conversion for the continuum in the narrow-band image

$$
F_{\mathrm{NB}}=\varepsilon N_{\mathrm{NB}} 2.1310^{-9} 10^{-0.4 R_{0}}
$$

The flux of a line emission at wavelength $\lambda_{i}$, where the filter has transmission $S_{i}$, is then given by:

$$
\begin{aligned}
F_{i} & =\varepsilon N(\mathrm{H} \alpha+[\mathrm{NII}]) 2.1310^{-9} 10^{-0.4 R_{0}} \mathrm{~W} \cdot\left(\langle\lambda\rangle / \lambda_{i}\right) \\
& \left(S_{\mathrm{peak}} / S_{i}\right) \mathrm{erg} \mathrm{s}^{-1} \mathrm{~cm}^{-2}
\end{aligned}
$$

where $W$ is the effective width of the narrow filter (i.e. the width of a rectangular filter with the same area and constant $S_{\text {peak }}$ throughput, $S_{\text {peak }}$ being the peak wavelength of the filter), and $\langle\lambda\rangle$ is the average wavelength of the filter. Actually, since both the $\mathrm{H} \alpha$ and the [NII] lines fall within the narrow-filter transmission curve, $S_{i}$ 
has been computed as the average of the filter transmissions in correspondence of the three lines, weighted by their intensity. For those galaxies with no measurement of the $[\mathrm{NII}] / \mathrm{H} \alpha$ ratios, we assumed $[\mathrm{NII}] \lambda 6584 / \mathrm{H} \alpha=1.38$ (see Sect. 4.2). Since narrow-band filters have boxy transmission functions, alternate choices for the lines ratios are likely to modify the computed flux by less than $10 \%$.

The $\mathrm{H} \alpha+[\mathrm{NII}]$ fluxes were then corrected for galactic extinction assuming $A_{6563} / E(B-V)=2.48$, and finally for redshift. No attempt was made to correct fluxes for internal extinction by dust. The luminosities $L_{\mathrm{H} \alpha+[\mathrm{NII}]}$ have been calculated using the distances derived from the Kraan-Korteweg (1986) Virgo infall model.

The observed fluxes, luminosities, linear diameters and morphology of the emitting regions are listed in Table 4. Also listed are the $\mathrm{H} \alpha /[\mathrm{NII}]$ ratios and the derived mass of the ionized gas (see Sect. 4.2).

The estimate of an upper limit for the flux is made difficult by the fact that the detection of a given amount of $\mathrm{H} \alpha+[\mathrm{NII}]$ emission depends on several factors such as its morphological distribution, the brightness of the continuum background, the photometric quality of the images, etc. Thus, we have calculated the upper limits in the following empirical way. Guided by the characteristics of the emission in galaxies with the faintest $\mathrm{H} \alpha+[\mathrm{NII}]$ fluxes, we assumed that a positive detection requires that the pixel values in the pure $\mathrm{H} \alpha+[\mathrm{NII}]$ image be significantly higher than the limiting flux in at least an area of about 100 pixels $\left(\sim 10 \operatorname{arcsec}^{2}\right)$. We therefore set the upper limits to be twice the limiting flux (determined from the noise measured in the pure $\mathrm{H} \alpha+[\mathrm{NII}]$ images) integrated over an area of one hundred pixels.

Our measurements are of course insensitive to diffuse gas distributed as the underlying stellar population, since its contribution would be absorbed in the scaling factor between narrow- and broad-band images. However, previous comparisons of the scaling factors with the theoretical expected values, computed from the filters efficiencies (TdSA), and with spectrophotometric measurements (GHJN), show that such component, if at all present, is very small.

\subsection{Comparison with previous measurements}

Some of the galaxies in our sample have previously measured fluxes (Trinchieri \& di Serego Alighieri 1991, hereinafter TdSA; S91; Hansen et al. 1991; Buson et al. 1993; GHJN; McMillan et al. 1994; Singh et al. 1995). Our results are compared with these previous determinations in Fig. 2; where available, the same aperture used by other observers has been used to measure our fluxes. S91 lists the integrated $\mathrm{H} \alpha+[\mathrm{NII}]$ flux in different apertures ranging from 0.5 to $2 \mathrm{kpc}$ in radius; for this comparison, we have chosen either the largest aperture with measured flux, or the smallest aperture available for those objects having only upper limits. This figure shows reasonable agreement (differences equal or less than a factor of 3 ) for most galaxies, with the exception of a few discrepant objects. The agreement seems to be better with TdSA and Buson et al. (1993) data than with GHJN and Singh et al. (1995). The magnitude of the scatter in $\mathrm{H} \alpha+[\mathrm{NII}]$ fluxes we find here is in line with the results from previous comparisons, see for instance Table 7 in GHJN and Fig. 7 in Buson et al. (1993). The most discrepant objects in Fig. 2 are: IC 1459, for which GHJN measure a flux much higher than our value and those published by S91 and Buson et al. (1993); NGC 720, where we find significant emission, in disagreement with both GHJN and S91, who did not detect any emission; NGC 1407, which has been "marginally detected" by GHJN; NGC 3607, for which Singh et al. (1995) measured a flux about five times larger than our value; NGC 1399, where there is a discrepancy of more than an order of magnitude between Singh et al. (1995) and GHJN. Among the objects in our sample not detected in $\mathrm{H} \alpha+[\mathrm{NII}]$, the only one with previous measurements is IC 4296, for which both GHJN and S91 find an emission concentrated in the inner $5^{\prime \prime}$. Unfortunately, our images (both $R$ and narrow-band) are saturated in the galaxy center, which therefore has prevented the detection of emission. The mean of the residuals (excluding upper limits) plotted in Fig. 2 is -0.03 , with a rms of 0.40 . Part of the scatter in the figure may possibly be due to systematic differences in the computation and calibration of previous data, and to the different flux thresholds in the various studies, which is very critical for galaxies with extended emission.

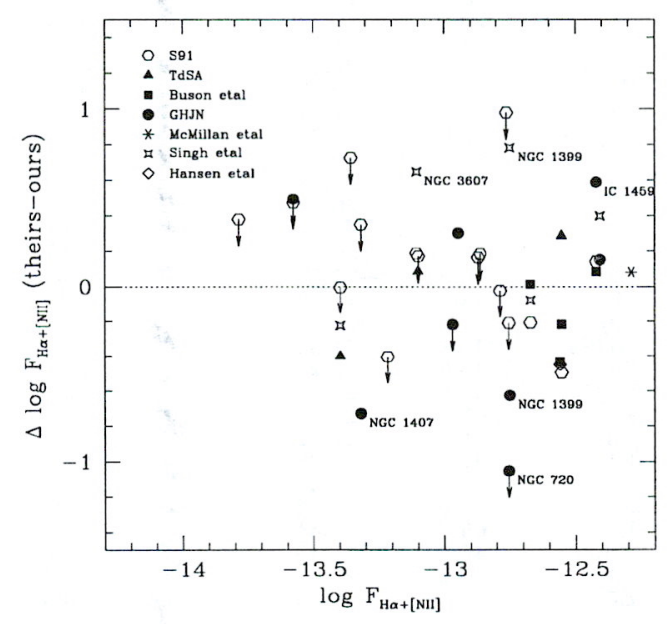

Fig. 2. Comparison of our $\mathrm{H} \alpha+[\mathrm{NII}]$ fluxes with the literature data 
Table 4. Data on our sample galaxies

\begin{tabular}{|c|c|c|c|c|c|c|c|c|c|c|c|}
\hline Ident. & Type & $\begin{array}{r}\text { Dist. } \\
(\mathrm{Mpc})\end{array}$ & $M_{B}$ & $10^{-14}$ & $\begin{array}{r}f_{\mathrm{H} \alpha+[\mathrm{NII}]} \\
\operatorname{erg~s} \mathrm{s}^{-1} \mathrm{~cm}^{-2} \\
\end{array}$ & $\begin{array}{r}L_{\mathrm{H} \alpha+[\mathrm{NII}]} \\
10^{39} \mathrm{erg} \mathrm{s}^{-1} \\
\end{array}$ & {$[\mathrm{NII}] \lambda 6584 / \mathrm{H} \alpha$} & $\log M_{\mathrm{HII}}$ & $\begin{array}{r}D_{\text {maj }} \\
\quad(k\end{array}$ & $\begin{array}{l}D_{\min } \\
\text { c) }\end{array}$ & Morph. \\
\hline NGC 524 & So/Sa & 44.1 & -22.05 & & 4.41 & 10.3 & - & 3.92 & 8.6 & 8.4 & $\mathrm{RE}$ \\
\hline NGC 533 & E3 & 96.7 & -22.71 & & 7.14 & 79.9 & - & 4.82 & 15.4 & 6.1 & $\mathrm{~F}$ \\
\hline NGC 584 & So & 33.2 & -21.40 & & 13.71 & 18.1 & - & 4.17 & 4.8 & 3.8 & $\mathrm{RE}$ \\
\hline NGC 596 & E0/So & 33.2 & -20.95 & & $<0.03$ & $<0.1$ & - & & - & - & - \\
\hline NGC 636 & $\mathrm{E} 1$ & 33.2 & -20.39 & & 3.64 & 4.8 & - & 3.60 & 1.2 & 0.6 & SD \\
\hline NGC 720 & E5 & 30.0 & -21.26 & & 17.66 & 19.1 & - & 4.19 & 5.0 & 2.5 & SD \\
\hline NGC 741 & E0 & 99.3 & -22.91 & & 2.45 & 28.9 & - & 4.38 & 11.3 & 5.4 & $\mathrm{RE}$ \\
\hline NGC 821 & E6 & 30.0 & -20.67 & & $<0.05$ & $<0.1$ & - & 4.00 & 11.0 & 0.4 & חE \\
\hline NGC 1172 & So & 27.1 & -19.60 & & 2.72 & 2.4 & - & 3.29 & 4.6 & 2.0 & $\bar{F}$ \\
\hline NGC 1399 & E1 & 26.2 & -21.65 & & 17.82 & 14.6 & - & 4.08 & 3.0 & 2.4 & SD \\
\hline NGC 1400 & So & 29.2 & -20.44 & & $<0.09$ & $<0.1$ & - & & - & - & - \\
\hline NGC 1407 & E0/S0 & 29.2 & -21.62 & & 4.82 & 4.9 & - & 3.60 & 3.5 & 2.9 & SD \\
\hline NGC 1453 & E0 & 69.9 & -21.96 & & 8.76 & 51.2 & - & 4.62 & 18.1 & 10.7 & $\mathrm{RE}$ \\
\hline NGC 1600 & $\mathrm{E} 4$ & 83.9 & -22.79 & & 4.01 & 33.9 & - & 4.44 & 11.7 & 4.9 & $\mathrm{~F}$ \\
\hline NGC 2865 & $\mathrm{E} 4$ & 48.4 & -21.24 & & $<0.15$ & $<0.5$ & - & - & 11.1 & - & - \\
\hline NGC 3115 & So & $\begin{array}{l}8.4 \\
8.7\end{array}$ & -19.97 & & $<0.22$ & $<0.1$ & 1.0 & - & 二 & 二 & Z \\
\hline NGC 3268 & $\mathrm{E} 2$ & 57.5 & -21.54 & & 0.36 & 1.4 & 1.0 & 3.16 & 1.9 & 1.9 & $\overline{\mathrm{SD}}$ \\
\hline NGC 3311 & S0 & 63.9 & -21.81 & & 0.53 & 2.6 & - & 3.33 & 1.5 & 1.5 & SD \\
\hline NGC 3379 & E0 & 14.5 & -20.62 & & 10.08 & 2.7 & - & 3.35 & 1.4 & 1.0 & $\mathrm{SD}$ \\
\hline NGC 3489 & $\mathrm{~S} 0 / \mathrm{Sa}$ & 9.2 & -18.66 & & 51.89 & 5.2 & - & 3.63 & 1.3 & 0.8 & $\mathrm{SD}$ \\
\hline NGC 3607 & So & 14.7 & -20.05 & & 7.83 & 2.0 & 二 & $\begin{array}{l}3.00 \\
3.22\end{array}$ & $\begin{array}{l}1.6 \\
1.6\end{array}$ & $\begin{array}{l}0.0 \\
0.9\end{array}$ & SD \\
\hline NGC 4105 & So & 34.9 & -21.38 & & 13.47 & 19.7 & - & 4.21 & 8.4 & 5.0 & RE \\
\hline NGC 4472 & E1/S0 & 21.3 & -22.31 & & 17.30 & 9.4 & - & 3.89 & 3.0 & 1.3 & $\mathrm{SD}$ \\
\hline NGC 4473 & $\mathrm{E} 5$ & 21.3 & -20.61 & & 2.65 & 1.4 & - & 3.07 & 1.2 & 0.9 & $\mathrm{SD}$ \\
\hline NGC 4486 & E0 & 21.3 & -22.15 & & 188.32 & 102.2 & - & $\begin{array}{l}. .01 \\
4.92\end{array}$ & 6.8 & 5.2 & $\mathrm{~F}$ \\
\hline NGC 4552 & So & 21.3 & -21.07 & & 7.96 & 4.3 & 1.3 & 3.57 & 1.6 & 1.1 & SD \\
\hline NGC 4636 & E0/So & 21.3 & -21.21 & & 21.37 & 11.6 & - & 3.98 & 3.1 & 2.3 & $\mathrm{SD}$ \\
\hline NGC 4782 & E0 & 82.6 & -22.03 & & 3.61 & 29.5 & - & 4.38 & 4.1 & 2.6 & $\mathrm{SD}$ \\
\hline NGC 4936 & $\mathrm{E} 2$ & 59.6 & -22.52 & & 13.05 & 55.5 & 1.9 & 4.66 & 3.7 & 2.3 & $\mathrm{SD}$ \\
\hline NGC 5044 & E0 & 52.2 & -21.92 & & 39.16 & 127.6 & & 4.93 & 18.9 & 11.5 & F \\
\hline NGC 5077 & So & 52.2 & -21.38 & & 16.29 & 53.1 & 0.7 & 4.64 & 12.0 & 5.6 & $\mathrm{RE}$ \\
\hline NGC 5090 & E2 & 60.1 & -21.92 & & 3.97 & 17.1 & & 4.33 & 1.7 & 1.2 & SD \\
\hline NGC 5102 & So & 3.0 & -17.38 & & 51.49 & 0.5 & - & 2.65 & 1.0 & 0.3 & SD \\
\hline NGC 5419 & So & 73.5 & $\begin{array}{l}-22.89 \\
\end{array}$ & & 4.14 & 26.7 & - & 4.34 & 4.4 & $\begin{array}{l}0.0 \\
2.9\end{array}$ & SD \\
\hline NGC 5532 & So & 133.3 & -22.85 & & 6.02 & 128.0 & - & 5.02 & 12.6 & 10.2 & $\mathrm{~F}$ \\
\hline NGC 5812 & E0 & 40.7 & -21.22 & & 5.83 & 11.5 & 1.3 & 3.98 & 2.1 & 2.1 & SD \\
\hline NGC 5813 & E1 & 34.9 & -21.30 & & 11.30 & 16.5 & - & 4.14 & 6.2 & 2.3 & $\mathrm{~F}$ \\
\hline NGC 5831 & $\mathrm{E} 4$ & 34.9 & -20.41 & & 2.29 & 3.4 & 1.8 & 3.44 & 1.2 & 0.4 & SD \\
\hline NGC 5846 & So & 34.9 & -21.81 & & 28.02 & 40.9 & - & 4.45 & 5.8 & 4.1 & RE \\
\hline NGC 5864 & SB0 & 33.9 & $\begin{array}{l}-21.01 \\
-20.02\end{array}$ & & 14.27 & $\begin{array}{l}40.9 \\
19.6\end{array}$ & 二 & 4.21 & $\begin{array}{r}0.0 \\
10.0\end{array}$ & $\begin{array}{l}4.1 \\
3.9\end{array}$ & $\mathrm{RE}$ \\
\hline NGC 5898 & so & 44.3 & -21.31 & & 16.38 & 38.5 & - & 4.50 & 8.0 & 7.4 & $\mathrm{RE}$ \\
\hline NGC 5903 & E3/S0 & 44.3 & -21.49 & & 5.21 & $\begin{array}{l}12.2 \\
\text { n }\end{array}$ & - & 4.00 & 5.3 & 3.5 & $\mathrm{RE}$ \\
\hline NGC 5920 & So & 265.6 & -22.75 & & $<0.20$ & $<13.8$ & - & - & - & - & - \\
\hline NGC 6034 & $\mathrm{E}$ ? & 205.1 & -22.18 & & $<0.10$ & $<4.0$ & - & - & - & - & - \\
\hline NGC 6305 & $\mathrm{E} / \mathrm{S} 0$ & 47.7 & -20.81 & & $<0.16$ & $<0.5$ & - & - & - & - & - \\
\hline NGC 6407 & $\mathrm{E}$ & 80.3 & -22.09 & & $<0.06$ & $<0.5$ & $\overline{1.2}$ & - & 二 & 二 & 二 \\
\hline NGC 6483 & $\stackrel{\mathrm{L}}{\mathrm{E}}$ & $\begin{array}{l}80.3 \\
85.2\end{array}$ & $\begin{array}{l}-22.09 \\
-22.02\end{array}$ & & $<0.06$ & $<0.5$ & $\begin{array}{l}1.2 \\
1.4\end{array}$ & 二 & 二 & 二 & 二 \\
\hline NGC 6721 & E1 & 76.7 & -21.74 & & 9.42 & 66.3 & 2.3 & $4 . \overline{78}$ & $\overline{7.2}$ & 5.9 & $\overline{\mathrm{RE}}$ \\
\hline NGC 6758 & $\mathrm{E} 2$ & 58.8 & -21.54 & & 6.83 & 28.2 & 1.8 & 4.38 & 12.2 & 7.1 & $\mathrm{RE}$ \\
\hline NGC 6776 & E1 pec & 94.4 & -22.16 & & 6.93 & 73.8 & 1.0 & 4.63 & 8.0 & 4.7 & $\mathrm{~F}$ \\
\hline NGC 6851 & $\mathrm{E} 4$ & 48.6 & -20.92 & & 5.19 & 14.7 & 1.9 & 4.00 & 4.1 & 4.9 & $\mathrm{RE}$ \\
\hline NGC 6861 & So & 48.6 & -21.51 & & 19.85 & 56.0 & 1.5 & 4.74 & $\begin{array}{l}4.1 \\
5.9\end{array}$ & 3.0 & $\mathrm{RE}$ \\
\hline NGC 6868 & E3/S0 & 48.6 & -21.94 & & 27.65 & 78.0 & 1.2 & 4.71 & 7.8 & $\begin{array}{l}0.0 \\
4.2\end{array}$ & $\mathrm{RE}$ \\
\hline NGC 6875 & So & 54.1 & -20.89 & & 2.59 & 9.1 & - & 3.84 & 5.0 & 3.5 & $\mathrm{RE}$ \\
\hline NGC 6876 & E3 & 67.9 & -22.33 & & $<0.27$ & $<1.5$ & - & - & - & - & - \\
\hline NGC 6909 & E5 & 48.6 & -20.92 & & $<0.03$ & $<0.1$ & - & - & 二 & 二 & 二. \\
\hline NGC 6936 & So & $\begin{array}{r}40.0 \\
110.6\end{array}$ & $\begin{array}{l}-21.70 \\
-20\end{array}$ & & 7.67 & $\begin{array}{r}0.1 \\
92.8\end{array}$ & 二 & $4 . \overline{88}$ & 15.0 & $\overline{4.6}$ & $\overline{\mathrm{F}}$ \\
\hline NGC 6964 & $\mathrm{E}$ pec & $\begin{array}{r}110.0 \\
70.8\end{array}$ & -20.87 & & $\begin{array}{l}1.01 \\
2.57\end{array}$ & $\begin{array}{l}92.8 \\
12.7\end{array}$ & 二 & $\begin{array}{l}4.88 \\
4.02\end{array}$ & $\begin{array}{r}15.0 \\
2.8\end{array}$ & $\begin{array}{l}4.6 \\
1.3\end{array}$ & $\stackrel{\mathrm{F}}{\mathrm{SD}}$ \\
\hline NGC 7014 & $\begin{array}{l}\mathrm{L}_{\mathrm{E}} \mathrm{pec} \\
\end{array}$ & 83.5 & $\begin{array}{l}-20.81 \\
-21.59\end{array}$ & & 13.25 & 110.5 & $\overline{1.1}$ & $\begin{array}{l}4.02 \\
4.96\end{array}$ & $\begin{array}{r}2.8 \\
14.7\end{array}$ & $\begin{array}{r}1.3 \\
11.7\end{array}$ & RE \\
\hline NGC 7029 & So & 49.4 & -21.05 & & 2.17 & 6.3 & 1.1 & $\begin{array}{l}4.90 \\
3.72\end{array}$ & 3.7 & 3.5 & SD \\
\hline NGC 7041 & So/E7 & 32.8 & -20.63 & & 3.59 & 4.6 & - & 3.58 & 5.6 & 2.9 & $\mathrm{RE}$ \\
\hline NGC 7049 & So/Sa & 38.1 & -21.34 & & 29.71 & 51.7 & - & 4.64 & 10.1 & 9.4 & $\mathrm{RE}$ \\
\hline NGC 7192 & So & $\begin{array}{l}38.1 \\
48.6\end{array}$ & $\begin{array}{l}-21.34 \\
-21.24\end{array}$ & & $\begin{array}{r}29.11 \\
7.79\end{array}$ & 22.0 & 二 & $\begin{array}{l}4.04 \\
4.33\end{array}$ & $\begin{array}{r}10.1 \\
8.7\end{array}$ & $\begin{array}{l}9.4 \\
7.7\end{array}$ & $\begin{array}{l}\mathrm{RE} \\
\mathrm{RE}\end{array}$ \\
\hline NGC 7196 & E3/S0 & $\begin{array}{l}40.0 \\
50.5\end{array}$ & $\begin{array}{l}-21.24 \\
-21.17\end{array}$ & & 3.39 & 10.3 & - & $\begin{array}{l}4.33 \\
3.93\end{array}$ & $\begin{array}{l}8.1 \\
5.5\end{array}$ & 4.2 & $\mathrm{RE}$ \\
\hline NGC 7562 & $\mathrm{E} 2$ & $\begin{array}{l}00.0 \\
61.3\end{array}$ & -21.57 & & $\begin{array}{l}. .39 \\
1.64\end{array}$ & 7.4 & $\overline{2.3}$ & $\begin{array}{l}3.95 \\
3.78\end{array}$ & $\begin{array}{l}0.0 \\
3.7\end{array}$ & $\begin{array}{l}4.2 \\
2.9\end{array}$ & SD \\
\hline NGC 7619 & E3 & 61.3 & -22.01 & & 6.09 & 27.4 & 2.1 & 4.35 & 2.8 & 1.3 & SD \\
\hline NGC 7626 & E1 & 61.3 & -21.88 & & $<0.20$ & $<0.9$ & & & - & & - \\
\hline NGC 7796 & $\mathrm{E} 1$ & 56.4 & -21.37 & & $<0.02$ & $<0.1$ & $\overline{1.8}$ & 二 & 二 & 二 & E \\
\hline IC 1459 & $\mathrm{E}$ & 29.0 & -21.48 & & 37.93 & 38.1 & - & 4.35 & 5.6 & 5.5 & $\overrightarrow{R E}$ \\
\hline IC 4296 & E0 & 65.0 & $\begin{array}{l}-21.48 \\
-22.64\end{array}$ & & $\begin{array}{r}31.93 \\
<0.06\end{array}$ & $\begin{array}{r}38.1 \\
<0.4\end{array}$ & 二 & 4.35 & 0.6 & 2.5 & LE \\
\hline IC 4797 & E pec & 46.6 & $\begin{array}{r}-21.49 \\
\end{array}$ & & $<0.29$ & $<0.8$ & 二 & 二 & 二 & 二 & E \\
\hline IC 4889 & So & 43.5 & -21.28 & & 9.48 & $\begin{array}{r}0.0 \\
21.4\end{array}$ & 二 & $4 . \overline{13}$ & $\overline{7.6}$ & $\overline{5.7}$ & $\overline{\mathrm{RE}}$ \\
\hline IC 5105 & E5 & 93.5 & -22.43 & & $\begin{array}{l}9.48 \\
2.36\end{array}$ & $\begin{array}{l}24.4 \\
24.7\end{array}$ & $\overline{1.3}$ & $\begin{array}{l}4.13 \\
4.31\end{array}$ & $\begin{array}{l}.00 \\
5.2\end{array}$ & $\begin{array}{l}5.1 \\
2.3\end{array}$ & $\mathrm{SD}$ \\
\hline
\end{tabular}

Notes to the table:

Ionized gas morphologies are coded as follows:

SD: small disk; RE: regular extended; F: filamentary.

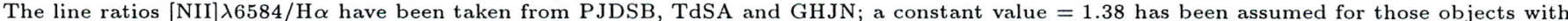

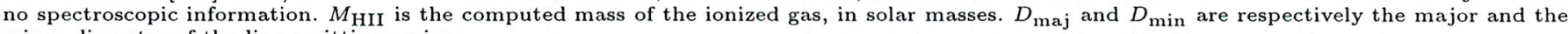
minor diameter of the line-emitting region. 


\section{Results}

\subsection{Ionized gas morphology}

A large fraction of $\mathrm{E}(72 \%)$ and $\mathrm{S} 0(85 \%)$ galaxies in our sample contain ionized gas; our rate of detection is larger than the results of previous imaging surveys (cf. $57 \%, \mathrm{GHJN})$, and spectroscopic surveys ( $\sim 50 \%$, PJDSB; $\sim 40 \%$, Caldwell 1984). The $\mathrm{H} \alpha+[\mathrm{NII}]$ luminosities range between $10^{38}-10^{41} \mathrm{erg} \mathrm{s}^{-1}$.

The ionized gas morphology appears to be rather smooth for most galaxies; however a significant fraction ( $\sim 12 \%$ of the galaxies), show a very extended filamentary structure. According to the morphology and size of the line-emitting region, the galaxies were classified into three broad classes:

1) small disk (SD): in some cases with quite faint and short filaments; the mean diameter is $\lesssim 4 \mathrm{kpc}$;

2) regular extended (RE): similar to the previous class, but larger in size ( 4 to $18 \mathrm{kpc}$ ), with short filaments sometimes present;

3) filaments (F): if a conspicuous filamentary structure dominates the morphology, often departing from a more regular disk-like inner region. It can extend as far as $10 \mathrm{kpc}$ from the galaxy center.

For each galaxy in our sample with detected emission, Figs. 9 displays the $\mathrm{H} \alpha+[\mathrm{NII}]$ emission (left panel) and the $R$ isophotes (right panel). North is up and East to the left for all images. For each object we give the lowest $\mathrm{H} \alpha+[\mathrm{NII}]$ contour level, which corresponds to the flux threshold level, in units of $10^{-16} \mathrm{erg} \mathrm{s}^{-1} \mathrm{~cm}^{-2} \operatorname{arcsec}^{-2}$, and the faintest $R$ isophotes, in $R$-mag $\operatorname{arcsec}^{-2}$. Contours are spaced $1 \mathrm{mag}$ apart. Also, we give the resolution (FWHM) of each emission-line image. All emission-line images have been slightly smoothed by convolution with a gaussian of $\sigma=1$ pixel.

The groups frequency distribution for our galaxies have been analyzed in order to correlate the gas morphology with the morphological type of the galaxy (E or S0; Fig. 3) and with its blue absolute magnitude $M_{B}$. Of all the S0 galaxies that show gas emission, $55 \%$ belong to the RE class, $34 \%$ to the SD class, and $11 \%$ to the filamentary class. The distribution for ellipticals is $25 \%$ for the RE, $54 \%$ for the SD, and $21 \%$ for the filamentary class. Therefore, elliptical galaxies that show SD gas structure are more frequent than those with $\mathrm{RE}$ structure, while it is the other way around for S0 galaxies. This is in line with the properties of stellar disks, which are both more luminous and larger in size in lenticular than in elliptical galaxies (see Scorza \& Bender 1995). No apparent correlation is however found between the boxyness parameter $a_{4}$ and either the luminosity or the size of the $\mathrm{H} \alpha+[\mathrm{NII}]$ emission. A detailed analysis of the correlations between the morphology and the kinematics of the ionized-gas and that of the stellar component will be the subject of a forthcoming paper.
There is no clear correlation between the gas morphology and the galaxy luminosity. While galaxies with filamentary structure tend to be more luminous, both in the optical bands and in $\mathrm{H} \alpha+[\mathrm{NII}]$, and those with small disks are generally fainter, at any given optical luminosity there is a range of about 1 dex in the mean diameter of the emitting region. On the other hand, the tighter correlation between the $\mathrm{H} \alpha+[\mathrm{NII}]$ luminosity and the size of the emitting region suggests that the $\mathrm{H} \alpha+[\mathrm{NII}]$ luminosity is mainly related to the extension of the gas distribution rather than to its projected brightness.

\subsection{Mass of ionized gas}

The total ionized gas mass can be derived from the $\mathrm{H} \alpha \mathrm{lu}-$ minosities under the assumption of case $\mathrm{B}$ recombination (Osterbrock 1974). We have derived the pure $\mathrm{H} \alpha$ luminosities using the [NII] $6584 / \mathrm{H} \alpha$ line ratios published in PJDSB, TdSA and GHJN, or adopting a constant value [NII] $6584 / \mathrm{H} \alpha=1.38$, that is the average found by PJDSB for their spectroscopic set of early-type galaxies (the ratio [NII] 6584/[NII] 6548 is kept constant $=3.0$, see PJDSB).

For a given electron temperature and density the mass can be written as:

$$
M(\mathrm{HII})=\left(L_{\mathrm{H} \alpha} m_{\mathrm{H}} / N_{\mathrm{e}}\right) /\left(4 \pi j_{\mathrm{H} \alpha} / N_{\mathrm{e}} N_{\mathrm{p}}\right)
$$

where $m_{\mathrm{H}}$ is the mass of the hydrogen atom; $N_{\mathrm{e}}$ and $N_{\mathrm{p}}$ are the number of electrons and protons per $\mathrm{cm}^{-3}$ and $j_{\mathrm{H} \alpha}$ is the emission coefficient of $\mathrm{H} \alpha$. Following PJDSB we have assumed an electron temperature of $10^{4} \mathrm{~K}$ and an electron density of $10^{3} \mathrm{e}^{-} \mathrm{cm}^{-3}$. The estimated masses are listed in Table 4 . The mass values range between $10^{3}$ and $10^{5}$ solar masses, in broad agreement with those derived by GHJN. Because of the range of line ratios displayed by early-type galaxies (depending on total luminosity and other galaxian parameters), and of the possible deviation of the gas temperature and density from the assumed values, these masses should be considered only as rough estimates.

\section{Correlations}

There have been previous attempts at determining the relationship between the hot $\left(10^{6}-10^{7} \mathrm{~K}\right)$ X-ray emitting gas and the warm $\left(\sim 10^{4} \mathrm{~K}\right) \mathrm{H} \alpha$-emitting gas. TdSA have found that on average galaxies with a larger content of hot gas also show a more powerful line emission; however, a large scatter in the relation indicates that other parameters must play a role.

The EINSTEIN sample of early-type galaxies (Fabbiano et al. 1992) have been analyzed statistically by Eskridge et al. (1995) to search for correlations between the X-ray luminosity and a variety of fundamental parameters of the galaxies. They found a significant correlation of the X-ray luminosities with the blue luminosities, the $\mathrm{X}$-ray color index $C_{12}$, the magnesium index $\mathrm{Mg}_{2}$ and the 
central velocity dispersion $\sigma_{0}$, in the sense that those systems with the deepest potential wells have the highest $L_{\mathrm{X}}$ and $\mathrm{Mg}_{2}$ values. Also, $L_{\mathrm{X}}$ correlates with the axial ratio $a / b$ : rounder galaxies tend to have higher $L_{\mathrm{X}}$ and $L_{\mathrm{X}} / L_{B}$. Galaxies with high $L_{\mathrm{X}}$ are usually boxy (i.e. with a negative $a_{4}$ coefficient; Bender et al. 1989). The blue luminosity is found to correlate with $a / b$ and the isophotal shape descriptor $a_{4}$, but not with the IRAS luminosities in either the $12 \mu \mathrm{m}$ and the $100 \mu \mathrm{m}$ bands. They suggest that it is the potential well that appears to govern both the ability to retain the ISM at the present epoch and to retain the enriched ejecta of early star formation bursts.

We have analyzed the possible relationships between the warm and the hot gaseous components of the ISM of the galaxies in our sample through the statistical correlation between the total $L_{\mathrm{H} \alpha}$ and $L_{\mathrm{X}}$ luminosities. The $\mathrm{X}$-ray fluxes for the sample galaxies have been taken from the EINSTEIN database (as published in Fabbiano et al. 1992), complemented by data taken from ROSAT observations published in Brinkmann et al. (1994). A significant correlation, though with a quite large scatter, between $\mathrm{H} \alpha+[\mathrm{NII}]$ and X-ray luminosities is found for those galaxies ( $27 \%$ of the sample) for which we have detected ionized gas and are listed as X-ray sources (see Fig. 4).

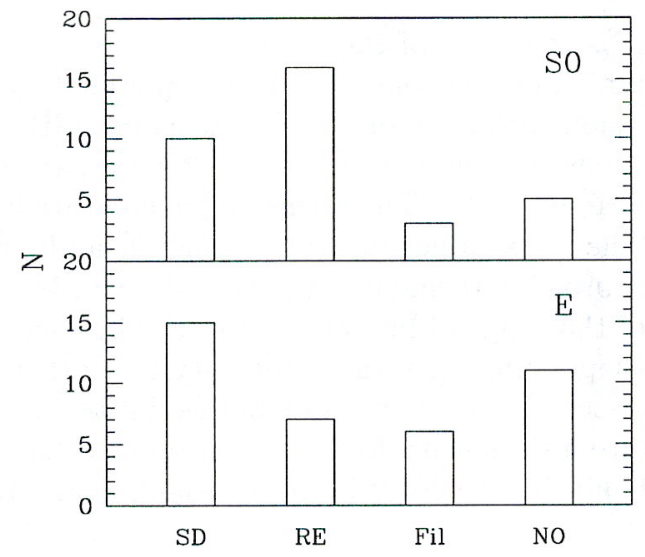

Fig. 3. Distribution of gas morphologies among ellipticals and among lenticulars. 'NO' means no detection

The strongest $L_{\mathrm{H} \alpha+[\mathrm{NII}]}$ galaxies also have strong $L_{\mathrm{X}}$ and present a more extended ionized gas distribution. However, among the remaining galaxies of the sample, there are relatively strong $\mathrm{X}$-ray emitting galaxies for which we have not detected $\mathrm{H} \alpha+[\mathrm{NII}]$ emission (for instance NGC 6034 and IC 4296) and objects which show emission-lines but are not listed either in the EINSTEIN or the ROSAT databases (for example NGC 6868 and NGC 7014). Upper limits for $L_{\mathrm{H} \alpha+[\mathrm{NII}]}$ and $L_{\mathrm{X}}$ are indicated in the figures by arrows. The distribution of the datapoints in the diagram shows that, while there is a
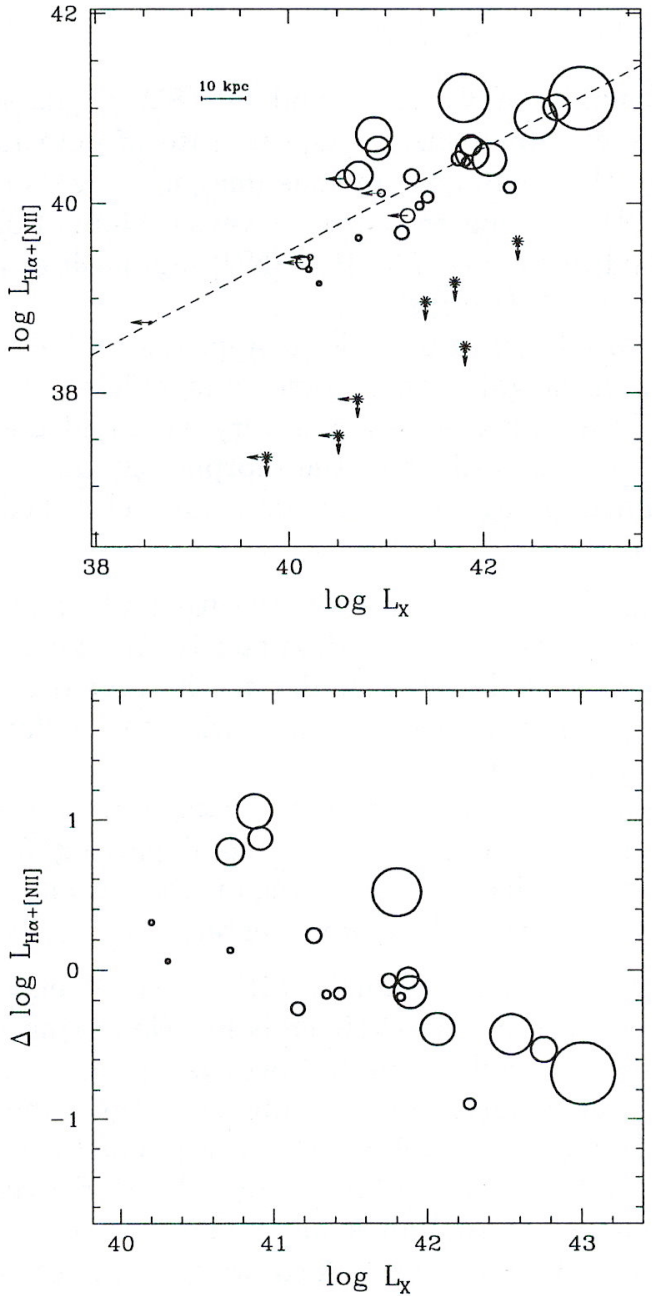

Fig. 4. Upper panel: $\mathrm{H} \alpha+[\mathrm{NII}]$ luminosities plotted against $\mathrm{X}$-ray luminosities. The size of each point is proportional to the size (mean diameter, i.e. $\sqrt{D_{\operatorname{maj}} \times D_{\min }}$ ) of the line-emitting region. Upper limits are marked with arrows. A least square fit to the datapoints gives a slope of 0.54. Lower panel: Residuals of $L_{\mathrm{H} \alpha+[\mathrm{NII}]}$ with respect to the best fitting line with slope 1

number of galaxies with significant X-ray luminosity but undetected in $\mathrm{H} \alpha+[\mathrm{NII}]$, the location of the upper limits on $L_{\mathrm{X}}$ alone is consistent with the general correlation between $L_{\mathrm{X}}$ and $L_{\mathrm{H} \alpha+[\mathrm{NII}]}$ exhibited by the galaxies with measured values in both quantities. Taken at face value, this seems to suggest that the presence of hot gas is a condition necessary but not sufficient for the presence of warm gas.

The lower panel of Fig. 4 shows, for those galaxies with positive detections in both X-ray and $\mathrm{H} \alpha+[\mathrm{NII}]$ emissions, the residuals of $L_{\mathrm{H} \alpha+[\mathrm{NII}]}$ with respect to the values computed assuming a direct relation (slope $=1$ ) between $L_{\mathrm{H} \alpha+[\mathrm{NII}]}$ and $L_{\mathrm{X}}$. It is evident that galaxies with higher $L_{\mathrm{X}}$ values have a relatively lower amount of ionized gas. 
The relationship between the warm gas and the farinfrared radiation produced by cold dust has been examined by comparing the $L_{\mathrm{H} \alpha+[\mathrm{NII}]}$ luminosities with $L_{\mathrm{FIR}}$ computed from the 60 and $100 \mu \mathrm{m}$ bands; no correlation has been found. (Infrared fluxes have been taken from Knapp et al. 1989). TdSA found no correlation between the masses of dust derived from IRAS infrared observation and the $\mathrm{H} \alpha+[\mathrm{NII}]$ luminosities. Nevertheless the physical association of dust and ionized gas in elliptical galaxies has been shown to exist from deep optical CCD imaging by GHJN. Figure 5 shows a correlation between the infrared luminosity in the $12 \mu \mathrm{m}$ band and $L_{\mathrm{H} \alpha+[\mathrm{NII}]}$. As in the correlation between $L_{\mathrm{H} \alpha+[\mathrm{NII}]}$ and $L_{\mathrm{X}}$ there seems to be galaxies with a significant $\mathrm{H} \alpha+$ [NII] luminosity but with only upper limits on the $12 \mu \mathrm{m}$ emission, and vice versa galaxies with significant infrared emission at this wavelength but little or no warm gas.

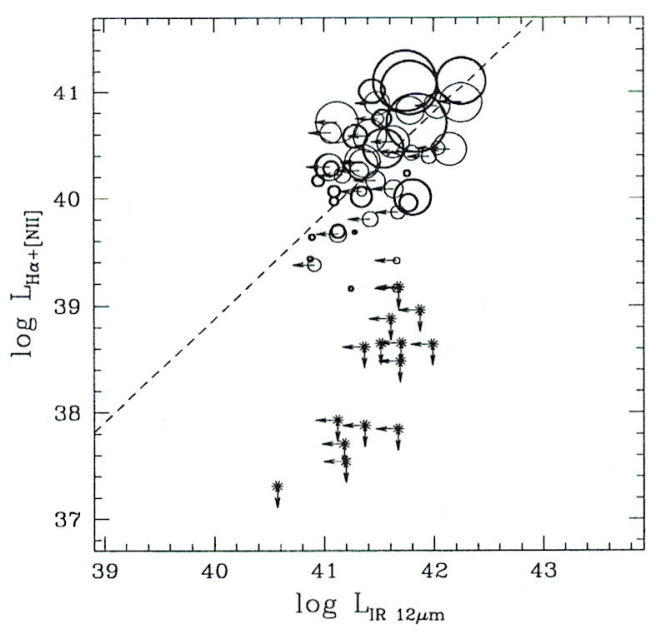

Fig. 5. $\mathrm{H} \alpha+[\mathrm{NII}]$ luminosities plotted against infrared luminosities in the $12 \mu \mathrm{m}$ band. Symbols as in the previous figure. The slope of the least-square fit to the datapoints (dashed line) is 0.97

No significant correlations were found with radio integrated and core luminosities (from Wrobel 1991 and Wrobel \& Heeschen 1991), although the intersection of our sample with the radio database is quite small and does not permit to draw any firm conclusion.

A weak correlation was found, for our galaxy sample, between $L_{\mathrm{H} \alpha+[\mathrm{NII}]}$ and the velocity dispersions $\sigma_{0}$, taken from the compilation by McElroy (1995) (Fig. 6). These correlations are in the sense that those systems with the deepest potential wells have the highest $\mathrm{H} \alpha+[\mathrm{NII}]$ luminosities. A similar result was found between $L_{\mathrm{X}}$ and $\sigma_{0}$. No correlation was found between $L_{\mathrm{H} \alpha+[\mathrm{NII}]}$ and $\mathrm{Mg}_{2}$ for the present sample. We have also looked for evidence of correlation between $\mathrm{H} \alpha+[\mathrm{NII}]$ luminosities and the merger history as parameterized by the "fine structure" index de- fined by Schweizer \& Seitzer (1992), finding no correlation for the 9 galaxies in common with Schweizer and Seitzer's sample.

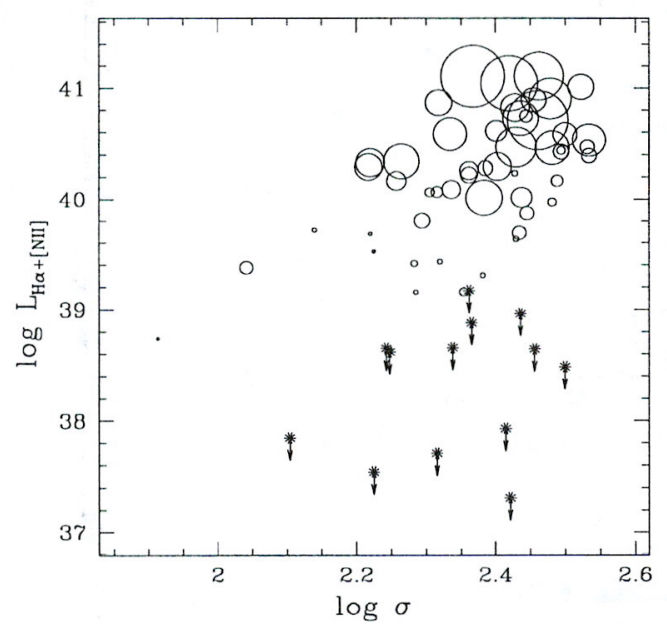

Fig. 6. Correlation between the central velocity dispersions and the $\mathrm{H} \alpha+[\mathrm{NII}]$ luminosities

Finally we have investigated the correlation between the $\mathrm{H} \alpha+[\mathrm{NII}]$ luminosities and the total blue luminosities of the galaxies (Fig. 7a). The weak trend present with total $B$ luminosities becomes much more evident when $L_{\mathrm{H} \alpha+[\mathrm{NII}]}$ is plotted versus the blue luminosity inside the region occupied by the line-emitting gas, $L_{B}(<$ emitt.) (Fig. 7b). We have defined $L_{B}(<$ emitt.) as the total luminosity inside a centered circular region with diameter equal to the mean diameter of the line-emitting region. This same result is obtained using fluxes instead of luminosities, which supports the fact that this is a real correlation, and not an artifact of selection effects (faint emission in nearby galaxies would be easier to detect than in more luminous and distant galaxies).

\section{Ionization mechanisms}

There are several potential mechanisms that can account in part or fully for the observed gas ionization. Among the competing theories are photoionization by hot young stars (Kim 1989, S91), by hot old stars (di Serego Alighieri et al. 1990, BMSB) or radiation originating in an active nucleus, and/or shock ionization (Heckman et al. 1989). Conductive heating by electrons associated with the $\mathrm{X}$ ray emitting coronal halo is another alternative proposed to explain the observed emission lines and their excitation character (SMG; Donahue \& Voit 1991). In this section we will test the applicability of some of these hypotheses to our observations and results. 

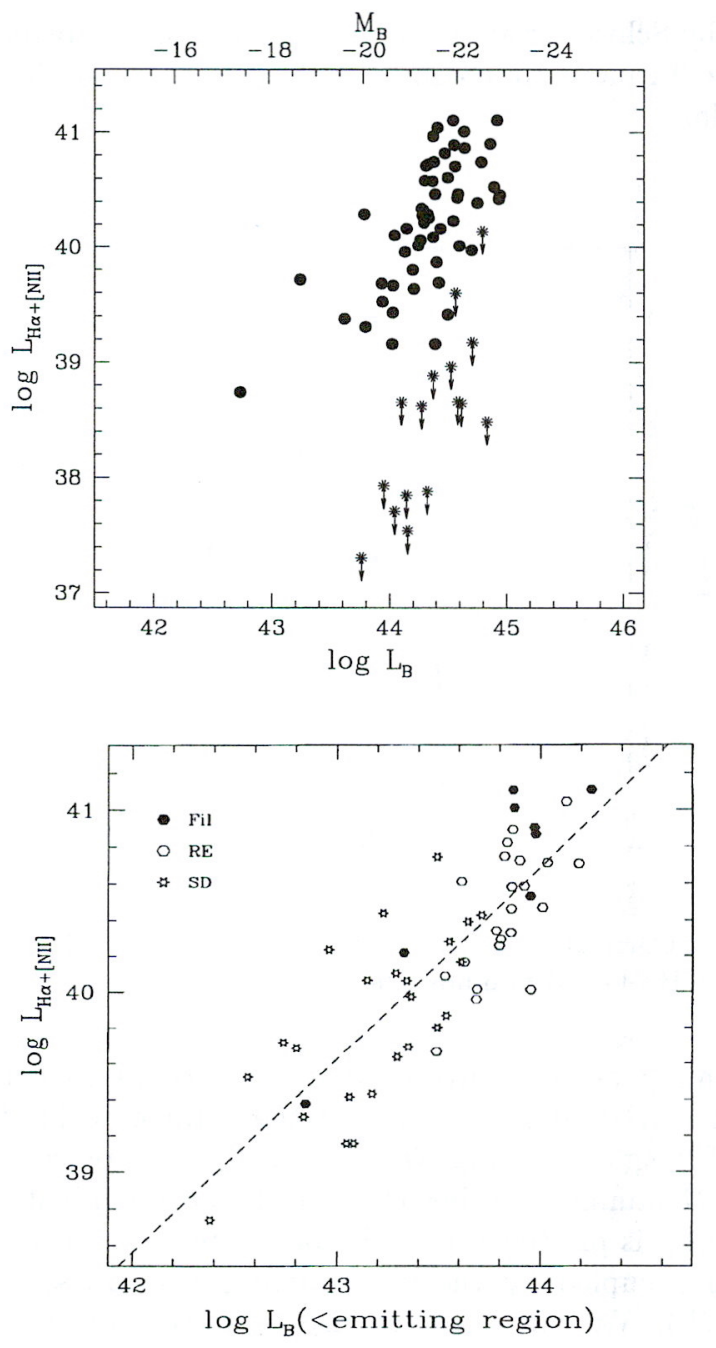

Fig. 7. The $\mathrm{H} \alpha+[\mathrm{NII}]$ luminosities are plotted against the total $B$ luminosities (upper panel) and the $B$ luminosities computed within the line-emitting region (lower panel). The slope of the least square fit (dashed line) is 1.06

\subsection{Photoionization by post-AGB stars}

The tight correlation between $L_{\mathrm{H} \alpha+[\mathrm{NII}]}$ and optical luminosity inside the emitting region strongly suggests a stellar origin for the ionizing photons. Recently BMSB have found that post-AGB stars provide sufficient ionizing radiation to account for the observed $\mathrm{H} \alpha$ luminosities and equivalent widths in very early-type galaxies. Following the prescriptions by BMSB, we have calculated for our galaxies the predicted $\mathrm{H} \alpha$ luminosities from stars in the post-AGB phase and compared them with the observed $\mathrm{H} \alpha$ luminosities.

According to the recombination theory, case B (Osterbrock 1974), $7.3410^{11}$ Lyman continuum photons are necessary to produce 1 erg of $\mathrm{H} \alpha$ luminosity. The total number of ionizing photons $Q(\mathrm{H})$ can be calculated assuming a specific photon luminosity of $7.310^{40}$ quanta/s $/ M_{\odot}$, and multiplying by the total mass of stars (in solar units) inside the emitting region, $M^{*}$; this in turn is derived from the blue luminosity $L_{B}$ inside the emitting region, assuming an integrated stellar mass-to-light ratio $M / L_{B}=8$ (BMSB).

Table 5 lists the blue luminosity, stellar masses, predicted number of ionizing photons, predicted $L_{\mathrm{H} \alpha}$ and the ratio between the predicted and observed $\mathrm{H} \alpha$ luminosities. We have found that for a large fraction of galaxies the ratios are of order unity, while several objects display values larger than 2, suggesting that the post-AGB stars provide a sufficient number of ionizing photons within the $\mathrm{H} \alpha$ emitting region. The agreement with the four galaxies in common with BMSB (NGC 4636, NGC 5846, NGC 6868 and IC 1459) is fair, considering the different gas luminosities and extensions we have found compared to their data. The presence of predicted/computed $\mathrm{H} \alpha$ luminosity ratios significantly larger than 1 is not an embarrassment, as there are several alternatives to explain an excess of available and unused photons. First, the covering factor of the gas can be below unity, as it is likely for ellipticals, which typically are not gas rich systems. In these cases, a significant fraction of extreme ultraviolet photons will escape to the intergalactic space, instead of being reprocessed by the interstellar medium. Post-AGB stars exist wherever there is an old stellar population, but it is not necessarily so for the gas, which, as observed, has a more complex and irregular distribution than the stellar component.

Another possible explanation is that some of the emitting clouds are not visible due to the large opacity of the dust along the line of sight. The galaxies in our sample have extensive dust systems, which are closely associated, at least morphologically, with the emission line gas. (We are currently completing the analysis of the dust characteristics, which will be the subject of a second paper in this series). It is possible that in the cases where there is significant amount of dust we calculate the correct value of $Q(\mathrm{H})$ from the blue luminosity, but we underestimate the $\mathrm{H} \alpha$ luminosity.

\subsection{Thermal conduction}

Conductive heating of the warm gas by hot electrons associated with an X-ray emitting coronal halo typically found within elliptical galaxies offers an alternative excitation mechanism, as proposed by Sparks \& CollierCameron (1988) and SMG. In this scenario, each galaxy has an associated pre-existing coronal halo, that is a diffuse hot gaseous plasma which pervades the galaxy. We then suppose that the galaxy accretes cold gas and dust either in a minor merger or as a result of a tidal encounter with a gas-rich companion. The process of thermal electron conduction then causes energy to flow from the hot coronal component into the cold gas which simultaneously excites the cold gas into emission while locally cooling the 
Table 5. Derived data on ionized gas

\begin{tabular}{|c|c|c|c|c|c|}
\hline Ident. & $\log L_{B}(\mathrm{emitt})$ & $\log M_{*}$ & $\log N_{\mathrm{ph}}$ & $\log L_{\mathrm{H} \alpha}$ & $L_{\mathrm{H} \alpha \text { calc }} / L_{\mathrm{H} \alpha \text { obs }}$ \\
\hline NGC 524 & 43.95 & 11.27 & 52.13 & 39.56 & 5.16 \\
\hline NGC 533 & 43.97 & 11.29 & 52.15 & 40.45 & 0.69 \\
\hline NGC 584 & 43.80 & 11.12 & 51.98 & 39.81 & 2.05 \\
\hline NGC 636 & 42.80 & 10.12 & 50.99 & 39.23 & 0.78 \\
\hline NGC 720 & 43.56 & 10.88 & 51.74 & 39.83 & 1.11 \\
\hline NGC 741 & 43.76 & 11.08 & 51.94 & 40.01 & 1.47 \\
\hline NGC 1172 & 42.85 & 10.17 & 51.03 & 38.92 & 1.75 \\
\hline NGC 1399 & 43.61 & 10.93 & 51.79 & 39.71 & 1.65 \\
\hline NGC 1407 & 43.35 & 10.67 & 51.53 & 39.24 & 2.67 \\
\hline NGC 1453 & 44.19 & 11.51 & 52.37 & 40.26 & 1.78 \\
\hline NGC 1600 & 43.95 & 11.27 & 52.14 & 40.08 & 1.56 \\
\hline NGC 3268 & 43.08 & 10.40 & 51.27 & 38.79 & 4.06 \\
\hline NGC 3311 & 43.06 & 10.38 & 51.25 & 38.96 & 2.63 \\
\hline NGC 3379 & 43.17 & 10.49 & 51.35 & 38.98 & 3.24 \\
\hline NGC 3489 & 42.74 & 10.06 & 50.92 & 39.26 & 0.62 \\
\hline NGC 3607 & 42.84 & 10.16 & 51.02 & 38.85 & 2.00 \\
\hline NGC 4105 & 43.81 & 11.13 & 51.99 & 39.84 & 1.92 \\
\hline NGC 4472 & 43.36 & 10.68 & 51.55 & 39.52 & 1.45 \\
\hline NGC 4473 & 43.05 & 10.37 & 51.23 & 38.70 & 4.58 \\
\hline NGC 4486 & 43.98 & 11.30 & 52.16 & 40.56 & 0.43 \\
\hline NGC 4552 & 43.30 & 10.62 & 51.48 & 39.20 & 2.60 \\
\hline NGC 4636 & 43.15 & 10.47 & 51.33 & 39.62 & 0.71 \\
\hline NGC 4936 & 43.50 & 10.82 & 51.68 & 40.29 & 0.33 \\
\hline NGC 5044 & 43.86 & 11.18 & 52.05 & 40.56 & 0.42 \\
\hline NGC 5077 & 43.90 & 11.22 & 52.08 & 40.27 & 0.88 \\
\hline NGC 5090 & 42.96 & 10.28 & 51.14 & 39.96 & 0.21 \\
\hline NGC 5102 & 42.38 & 9.70 & 50.56 & 38.28 & 2.59 \\
\hline NGC 5419 & 43.71 & 11.03 & 51.90 & 39.97 & 1.14 \\
\hline NGC 5532 & 44.25 & 11.57 & 52.43 & 40.65 & 0.82 \\
\hline NGC 5812 & 43.34 & 10.66 & 51.53 & 39.61 & 1.12 \\
\hline NGC 5813 & 43.41 & 10.73 & 51.59 & 39.78 & 0.74 \\
\hline NGC 5831 & 42.56 & 9.88 & 50.75 & 39.07 & 0.65 \\
\hline NGC 5846 & 43.54 & 10.86 & 51.72 & 40.08 & 0.71 \\
\hline NGC 5898 & 43.92 & 11.24 & 52.10 & 40.13 & 1.28 \\
\hline NGC 5903 & 43.53 & 10.85 & 51.71 & 39.63 & 1.64 \\
\hline NGC 6721 & 43.84 & 11.16 & 52.02 & 40.42 & 0.55 \\
\hline NGC 6758 & 44.01 & 11.33 & 52.19 & 40.01 & 2.07 \\
\hline NGC 6776 & 43.97 & 11.29 & 52.16 & 40.26 & 1.08 \\
\hline NGC 6851 & 43.63 & 10.95 & 51.81 & 39.63 & 2.07 \\
\hline NGC 6861 & 43.84 & 11.16 & 52.02 & 40.38 & 0.59 \\
\hline NGC 6868 & 43.87 & 11.19 & 52.05 & 40.34 & 0.70 \\
\hline NGC 6875 & 43.69 & 11.01 & 51.87 & 39.47 & 3.38 \\
\hline NGC 6964 & 43.37 & 10.69 & 51.55 & 39.65 & 0.90 \\
\hline NGC 7014 & 44.13 & 11.45 & 52.31 & 40.59 & 0.71 \\
\hline NGC 7029 & 43.50 & 10.82 & 51.68 & 39.35 & 2.92 \\
\hline NGC 7041 & 43.49 & 10.81 & 51.67 & 39.21 & 3.94 \\
\hline NGC 7049 & 44.03 & 11.36 & 52.22 & 40.27 & 1.21 \\
\hline NGC 7192 & 43.78 & 11.11 & 51.97 & 39.96 & 1.39 \\
\hline NGC 7196 & 43.69 & 11.01 & 51.87 & 39.56 & 2.80 \\
\hline NGC 7562 & 43.54 & 10.86 & 51.72 & 39.41 & 2.77 \\
\hline NGC 7619 & 43.23 & 10.55 & 51.41 & 39.98 & 0.36 \\
\hline IC 1459 & 43.86 & 11.18 & 52.04 & 39.98 & 1.56 \\
\hline IC 4889 & 43.85 & 11.18 & 52.04 & 39.76 & 2.60 \\
\hline IC 5105 & 43.64 & 10.96 & 51.83 & 39.94 & 1.05 \\
\hline
\end{tabular}

Notes to the table:

$\log L_{B}$ (< emitt.) is the $B$-band luminosity inside the line-emitting region; $\log M_{*}$ is the corresponding stellar mass (assuming $M / L=8$ ); $\log N_{\mathrm{ph}}$ is the number of ionizing photons; $\log L_{\mathrm{H} \alpha}$ is the "pure" H $\alpha$ luminosity, computed from $L_{\mathrm{H} \alpha+[\mathrm{NII}]}$ and the line ratio $[\mathrm{NII}] \lambda 6584 / \mathrm{H} \alpha$. The last column gives the ratio between the predicted and the computed H $\alpha$ luminosity. 
coronal gas. The energy radiated by the cold gas (the optical emission filament system in this picture) is simply the heat flux into the filaments. Quantitatively, this idea has worked well with previously studied objects and explains the dustiness of the gas as well as the X-ray spectral parameters (Sparks 1992). Here, we investigate whether such a mechanism is at all viable as a general excitation process for emission line gas in ellipticals.

Under the assumption that the available energy flux is in the saturated regime (Cowie \& McKee 1977), the amount of energy available to excite the optical emission filaments is, following $\mathrm{SMG}, Q_{\text {sat }}=$ $5.210^{40} T_{7}^{3 / 2} n_{0.01} D_{\text {maj }} D_{\text {min }}$, where $T_{7}=T / 10^{7}$ is the temperature of the coronal halo and $n_{0.01}=n_{\mathrm{e}} / 0.01$ is its density. In terms of the observed emission line flux, that is

$$
L_{\mathrm{H} \alpha+[\mathrm{NII}]}(\text { predicted })=1.510^{39} T_{7}^{3 / 2} n_{0.01} D_{\text {maj }} D_{\text {min }}
$$

assuming that $\sim 1 \%$ of the available energy is radiated in the $\mathrm{H} \alpha+[\mathrm{NII}]$ lines and that the total surface area of the system is three times the apparent projected surface area.

The average coronal gas temperature for elliptical galaxies found by Kim et al. (1992) is $T \approx 1.5 \mathrm{keV}$, while characteristic densities for the coronal gas in elliptical galaxies are $n_{\mathrm{e}} \approx 0.01 \mathrm{~cm}^{-3}$. With these values for the temperature and density, we predict a filament luminosity and show the comparison to observations in Fig. 8.

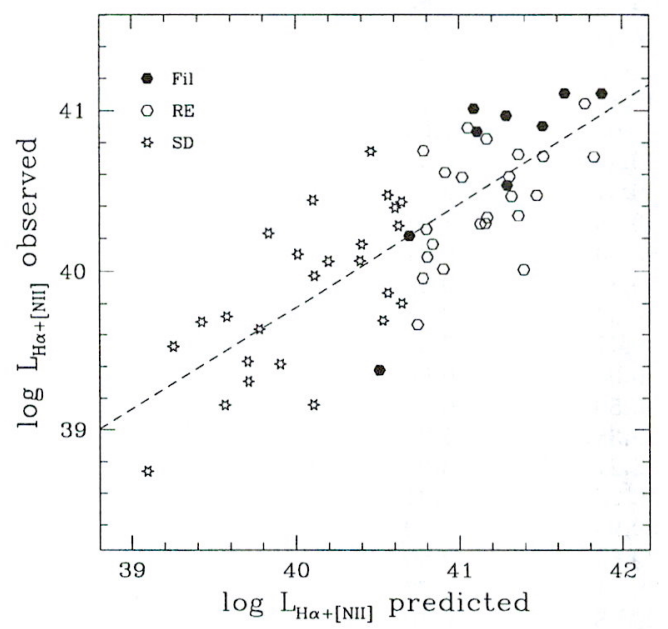

Fig. 8. The observed $\mathrm{H} \alpha+[\mathrm{NII}]$ luminosity is plotted versus the luminosity predicted according to the simple model of conductive heating of the warm gas by hot electrons emitted from the X-ray halo. The slope of least square fit (dashed line) is 0.64 , the $\mathrm{rms}$ is 0.33

No account has yet been taken of the variation of coronal gas parameters such as temperature and density with galaxy size or mass, nor has any adjustment made to the absolute normalization of SMG. The degree of correlation and the absolute level of the correlation are excellent, and demonstrate that an interpretation based on mergers and thermal interaction of hot and cold plasma components at this stage is perfectly consistent with the data.

\section{Summary}

We have shown the results of an optical imaging survey of 73 luminous early-type galaxies directed to study the properties of their interstellar medium. The galaxies were selected to span the entire variety of morphological types and isophotal shapes from E0 through $\mathrm{S} 0 / \mathrm{Sa}$, to provide a fair interval of optical luminosity, to include both radioloud and radio-quiet, $\mathrm{X}$-ray emitters and non-emitters

From our observations, which consisted of broad-band $R$ and narrow-band images centered at the $\mathrm{H} \alpha$ and [NII] emission lines, we have derived and mapped the luminosity and morphological distribution of the ionized gas. We have found that a large fraction of $\mathrm{E}(72 \%)$ and $\mathrm{S} 0(85 \%)$ galaxies in our sample contain measurable amounts of ionized gas. This high rate of detection and the measured integrated $\mathrm{H} \alpha+[\mathrm{NII}]$ fluxes are larger than those derived by previous studies. This is due, to a great extent, to the longer exposures, higher signal-to-noise ratio and therefore lower limiting fluxes in our sample when compared to other studies. This is apparent when direct comparison are made of the extended, faint filamentary structures seen in our data and those of previous studies.

When comparing the properties of the ionized gas with the morphological type of the galaxy, we find that the ellipticals have twice as many instances of extended filamentary structures (21\%) than the S0 galaxies, but more than half $(64 \%)$ of the ellipticals have emission concentrated in small disks. This is in line with the properties of stellar disks which are both more luminous and larger in size in lenticular than in elliptical galaxies. While there is at best a weak correlation between the extent of the filamentary structure and the overall galaxy luminosity, we find a tighter correlation between the $\mathrm{H} \alpha+[\mathrm{NII}]$ luminosity and the size of the emitting region. This indicates that the $\mathrm{H} \alpha+[\mathrm{NII}]$ luminosity is mainly related to the gas extension rather than to its projected brightness.

We have estimated the total mass of ionized gas for each galaxy, assuming that the case $\mathrm{B}$ recombination conditions are applicable, and an electron temperature of $10^{4}$ $\mathrm{K}$ and density of $10 \mathrm{e}^{-} \mathrm{cm}^{-3}$. With these obviously rough assumptions, we derive total masses for the ionized gas in the range of $10^{3}-10^{5} M_{\odot}$, well in line with previous results. To improve the accuracy of these results, as well as to derive kinematical information on the most interesting emission-line objects, we are carrying-out a program of long-slit spectroscopy, whose outcome will be the subject of a future publication.

We have explored the possible correlation between the hot $\left(10^{6}-10^{7} \mathrm{~K}\right) \mathrm{X}$-ray emitting gas and the warm $\left(10^{4} \mathrm{~K}\right)$ 
$\mathrm{H} \alpha$-emitting component. For those galaxies in our sample which are both X-ray emitters and show the presence of ionized gas, we find a significant correlation between the $\mathrm{X}$-ray and $\mathrm{H} \alpha+[\mathrm{NII}]$ luminosities. However among the remaining galaxies of the sample there are relatively strong $\mathrm{X}$-ray emitters for which we have not detected $\mathrm{H} \alpha+[\mathrm{NII}]$ emission, and vice versa $\mathrm{H} \alpha+[\mathrm{NII}]$ emitters that are not listed in the catalogs of X-ray sources. The distribution of upper limits in the $L_{\mathrm{H} \alpha+[\mathrm{NII}]}-L_{\mathrm{X}}$ diagram suggests that warm gas is only found in galaxies with a substantial amount of hot gas, while the presence of hot gas does not necessarily imply that of the warm component.

We have estimated the emission-line flux under the assumption that the excitation mechanism is conductive heating of the warm gas by hot electrons associated with an X-ray emitting coronal halo. The predicted $\mathrm{H} \alpha+[\mathrm{NII}]$ luminosity correlates extremely well with the measured values. We must note however that this correlation holds even for those galaxies where there is no measured $\mathrm{X}$ ray emission, and therefore it cannot be construed as a proof that X-ray conduction by hot electrons is necessarily the only mechanism responsible for the observed lineemission.

This has an important bearing on our understanding of the mechanisms responsible for the heating and ionization of the hot and warm gas, and whether we should invoke a single mechanism, and therefore expect strong a correlation between the two gas phases, or seek separate but somehow related mechanisms, and therefore expect weaker correlations. This issue is still very much open and will require further observations, as well as additional theoretical work.

A reasonable correlation was found between the $\mathrm{H} \alpha+[\mathrm{NII}]$ luminosities and the infrared IRAS $12 \mu \mathrm{m} \mathrm{lu}-$ minosities, but no correlation was found with the $60 \mu \mathrm{m}$ and $100 \mu \mathrm{m}$ luminosities.

We found weak correlation between the $\mathrm{H} \alpha+[\mathrm{NII}]$ luminosity and the velocity dispersion of the galaxy, in the sense that those systems with the deepest potential wells have the highest emission-line luminosities. On the other hand, there is a strong correlation between the blueluminosities within the emitting region and the emissionline luminosity. This is an important result which shows that the source of the ionizing photons is distributed in the same way as the stellar population.

A mechanism with the necessary characteristics is that proposed by BMSB, who found that post-AGB stars provide sufficient ionizing radiation to account for the observed $\mathrm{H} \alpha$ luminosities and equivalent widths in earlytype galaxies. For our galaxies, we have calculated the predicted $\mathrm{H} \alpha$ luminosities produced by stars in the postAGB phase and compared them with the observed $\mathrm{H} \alpha$ luminosities. We find that in all cases the ratios are of the order of unity or greater, meaning that the post-AGB stars can produce all the necessary ionizing photons and are distributed as required to effectively produce the $\mathrm{H} \alpha$ fluxes. In several cases there is even a remarkable excess of ionizing photons, which are then probably lost to the galaxy because of covering factors less than unity. The post-AGB stars are therefore a very plausible and attractive ionization mechanism to explain the observed $\mathrm{H} \alpha$ luminosity. We know that they do exist, are distributed in the same way as the rest of the old stellar population in the galaxy and therefore can account naturally for several of the observed correlations (eg. with the blue luminosity, with the size of the emitting region, with the velocity dispersion, etc.). However, the observed correlation with the $\mathrm{X}$-ray fluxes cannot be directly explained by the presence of post-AGB stars, and a different mechanism must be invoked. More extensive observations, in particular long-slit spectroscopy, as well as more work on the theoretical modeling of the mechanisms responsible for the X-ray flux, are clearly needed.

Acknowledgements. These observations were obtained at the ESO telescopes in La Silla, within the ESO Key-Programme 1-004-43K. We thank the ESO staff for their support. N. Caon acknowledges the receipt of an ESA Fellowship. M. Pastoriza acknowledges the support and hospitality of the STScI during a sabbatical visit. We dedicate this paper to the memory of our good friend and colleague, Jean-Luc Nieto, who was one of the leaders in the field of early-type galaxies and a member of our Key-Programme team.

\section{References}

Bender R., Surma P., Döbereiner S., Möllenhoff C., Madejsky R., 1989, A\&A 217, 35

Binette L., Magris C.G., Stasins̀ka G., Bruzual A.G., 1994, A\&A 292, 13 (=BMSB)

Brinkmann W., Siebert J., Boller Th., 1994, A\&A 281, 355

Buson L.M., Sadler E.M., Zeilinger W.W., et al., 1993, A\&A 280, 409

Caldwell N., 1984, PASP 96, 287

Cowie L.L., McKee C.F., 1977, ApJ 211, 135

Davies R.L., Burstein D., Dressler A., et al., 1987, ApJS 64, 581

de Vaucouleurs A., Longo G., 1987, The Univ. of Texas Monographs in Astron., 5

de Vaucouleurs G., de Vaucouleurs A., Corwin H.G. Jr., et al., 1991, Third Reference Catalogue of Bright Galaxies. Springer-Verlag, New York

di Serego Alighieri S., Trinchieri G., Brocato E. 1990, in: Fabbiano G., Gallagher J.S., Renzini A. (eds.), Windows on Galaxies. Kluwer, Dordrecht, p. 301

Donahue M., Voit G.M., 1991, ApJ 381, 361

Eskridge P.B., Fabbiano G., Kim D.-W., 1995, ApJ 442, 523

Fabbiano G., Kim D.-W., Trinchieri G., 1992, ApJS 80, 531

Fabian A.C., 1994, ARAA 32, 277

Fosbury R.A.E., Boksenberg A., Snijders M.A.J., et al., 1982, MNRAS 201, 991

Goudfrooij P., Hansen L., Jørgensen H.E., Nørgaard-Nielsen H.U., 1994, A\&AS 105, 341 (=GHJN) 
Hansen L., Jørgensen H.E., Nørgaard-Nielsen H.U., 1991, A\&A 243, 49

Heckman T., Baum S.A., vanBreugel W.J.M., McCarthy P., 1989, ApJ 338, 48

Jerjen H., Tammann G., 1993, A\&A 276, 1

Kim D.-W., 1989, ApJ 346, 653

Kim D.-W., Fabbiano G., Trinchieri G., 1992, ApJ 393, 134

Knapp G.R., Guhathakurta P., Kim D.-W., Jura M., 1989, ApJS 70, 387

Kraan-Korteweg R.C., 1986, A\&AS 66, 255

Macchetto F., Sparks W.B., 1992, in: Busarello G., Capaccioli M., Longo G. (eds.), Morphological and Physical Classification of Galaxies. Kluwer, Dordrecht, p. 191

McElroy D., 1995, ApJS 100, 105

McMillan R., Ciardullo R., Jacoby G.H., 1994, AJ 108, 1610

Osterbrock D.E., 1974, Astrophysics of Gaseous Nebulae. W.H. Freeman \& Co., San Francisco

Phillips M.M., Jenkins C.R., Dopita M.A., Sadler E.M., Binette L., 1986, AJ 91, 1062 (=PJDSB)
Poulain P., Nieto J.-L., 1994, A\&AS 103, 573

Sadler E.M., Gerhard O.E., 1985, MNRAS 214, 177

Sandage A.R., Tammann G., 1987, (a revised) Shapley-Ames

Catalog of Bright Galaxies (2nd ed.). Carnegie, Washington Schweizer F., Seitzer P., 1992, AJ 104, 1039

Scorza C., Bender R., 1995, A\&A 293, 20

Shields J., 1991, AJ 102, 1314 (=S91)

Singh K.P., Bhat P.N., Prabhu T.P., Kembhavi A.K., 1995, A\&A 302, 658

Sparks W.B., 1992, ApJ 399, 66

Sparks W.B., Collier-Cameron A., 1988, MNRAS 232, 215

Sparks W.B., Macchetto F., Golombek D., 1989, ApJ 345, 153 (=SMG)

Sparks W.B., Wall J.V., Thorne D.J., et al., 1985, MNRAS 217,87

Trinchieri G., di Serego Alighieri S., 1991, AJ 101, 1647 $(=\mathrm{TdSA})$

Wrobel J.M., 1991, AJ 101, 127

Wrobel J.M., Heeschen D.S., 1991, AJ 101, 148 

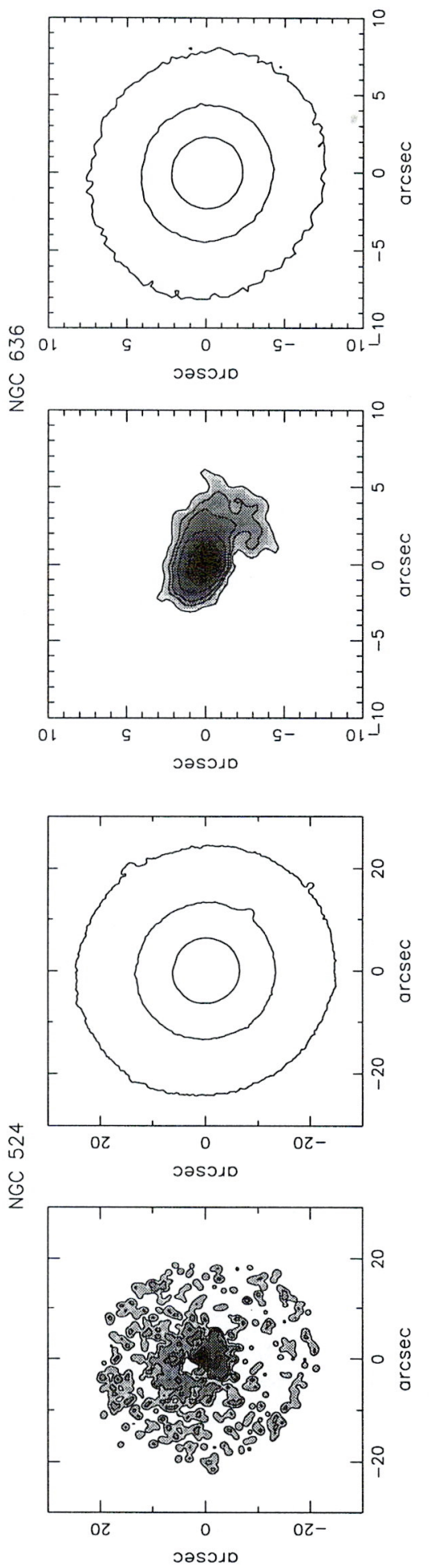
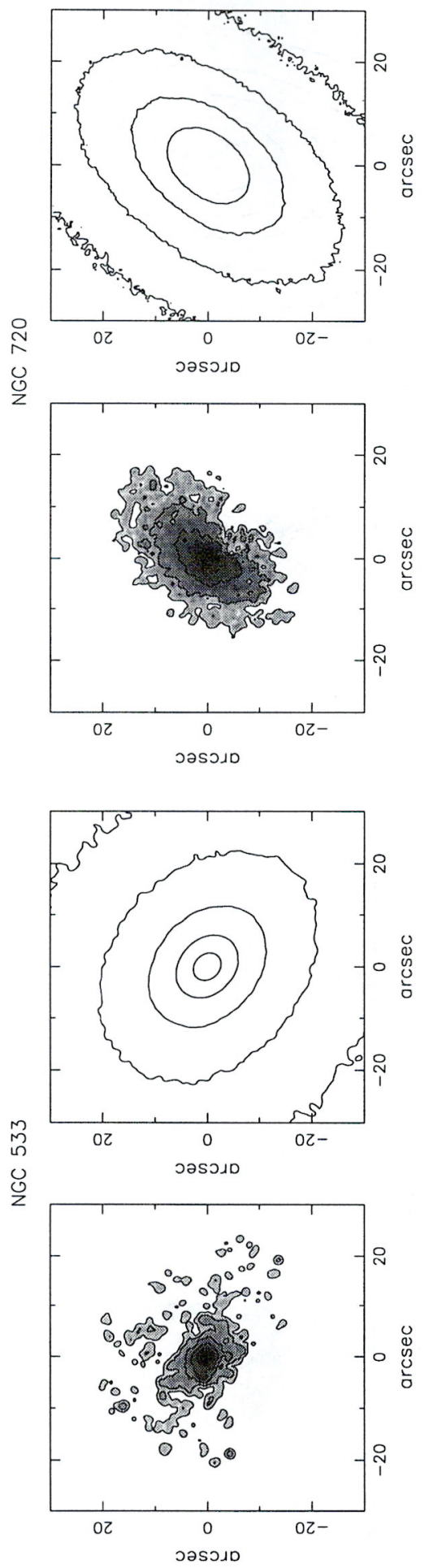
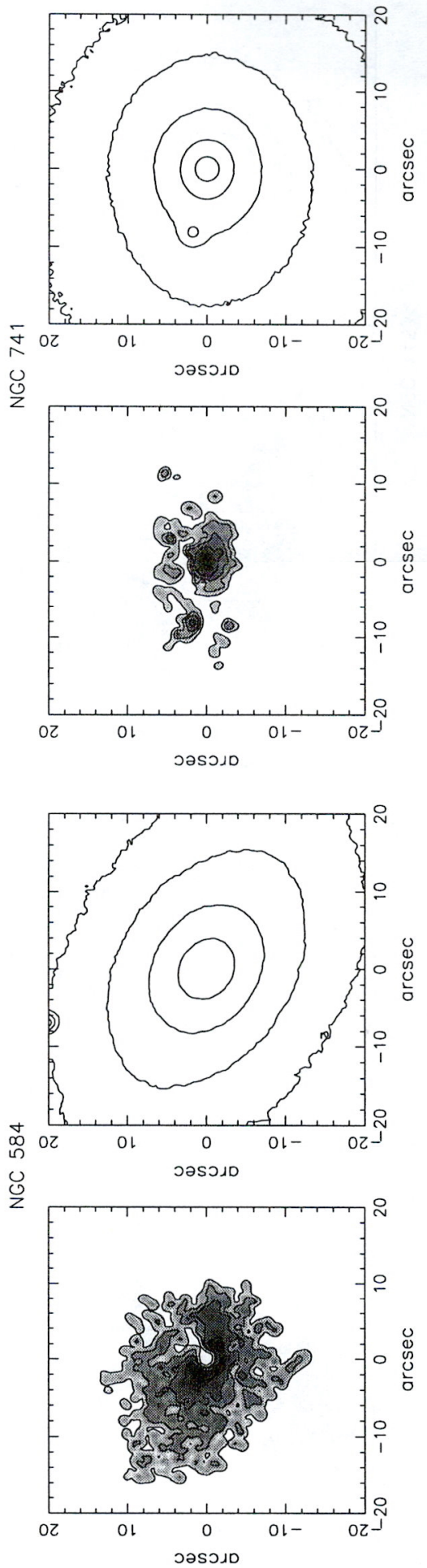

Fig. 9. NGC 524. $\mathrm{H} \alpha+[\mathrm{NII}]: 0.2 ; R: 22.0 ; \mathrm{FWHM}=1$." $4 ; \quad$ NGC 533. $\mathrm{H} \alpha+[\mathrm{NII}]: 0.3 ; R: 22.0 ; \mathrm{FWHM}=1{ }^{\prime \prime} 5 ; \quad$ NGC 584. $\mathrm{H} \alpha+[\mathrm{NII}]: 0.5 ; R: 20.0 ; \mathrm{FWHM}=1{ }^{\prime \prime} 3 ; \quad$ NGC 636. $\mathrm{H} \alpha+[\mathrm{NII}]: 0.7 ; R: 19.0 ; \mathrm{FWHM}=1{ }^{\prime \prime} 7 ; \quad$ NGC 720. H $\alpha+[\mathrm{NII}]: 0.8 ; R$ : 21.0; FWHM=1." $6 ; \quad$ NGC 741. $\mathrm{H} \alpha+[\mathrm{NII}]: 0.3 ; R: 21.0 ; \mathrm{FWHM}=1 . " 4$ 

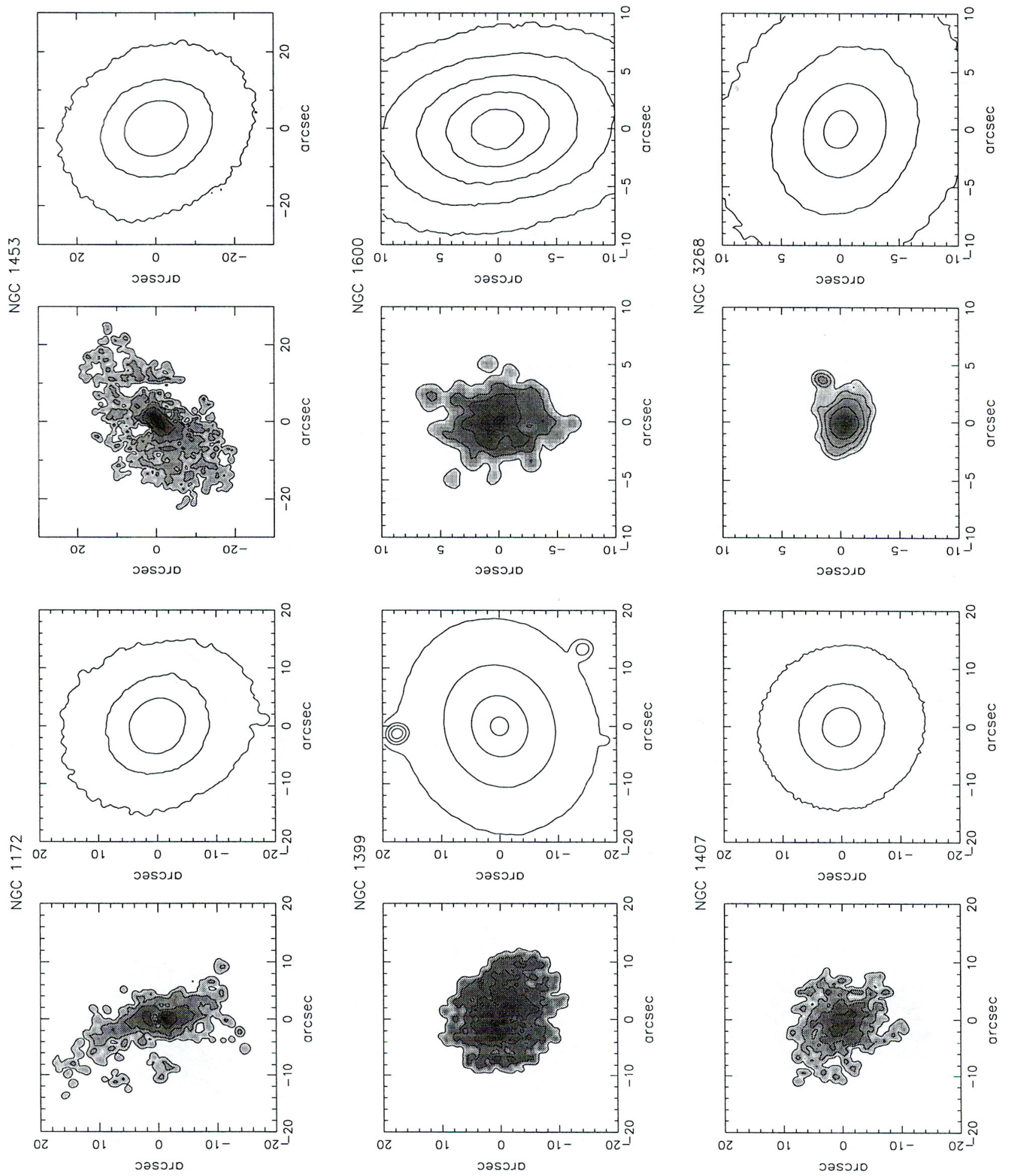

Fig. 9. NGC 1172. $\mathrm{H} \alpha+[\mathrm{NII}]: 0.25 ; R: 21.0 ; \mathrm{FWHM}=1 .{ }^{\prime \prime} 2 ; \quad \mathrm{NGC}$ 1399. H $\alpha+[\mathrm{NII}]: 1.0 ; R: 19.0 ; \mathrm{FWHM}=1 .^{\prime \prime} 4 ; \quad \mathrm{NGC}$ 1407. $\mathrm{H} \alpha+[\mathrm{NII}]: 0.4 ; R: 19.0 ; \mathrm{FWHM}=1 .{ }^{\prime \prime} 3 ; \quad \mathrm{NGC}$ 1453. $\mathrm{H} \alpha+[\mathrm{NII}]: 0.2 ; R: 21.0 ; \mathrm{FWHM}=1 .{ }^{\prime \prime} 7 ; \quad \mathrm{NGC} \mathbf{1 6 0 0} . \mathrm{H} \alpha+[\mathrm{NII}]:$ $0.5 ; R: 19.5$ (step=0.5 mag); FWHM=1." $3 ; \quad$ NGC 3268. H $\alpha+[\mathrm{NII}]: 0.1 ; R: 20.0 ; \mathrm{FWHM}=1 .{ }^{\prime \prime} 1$ 

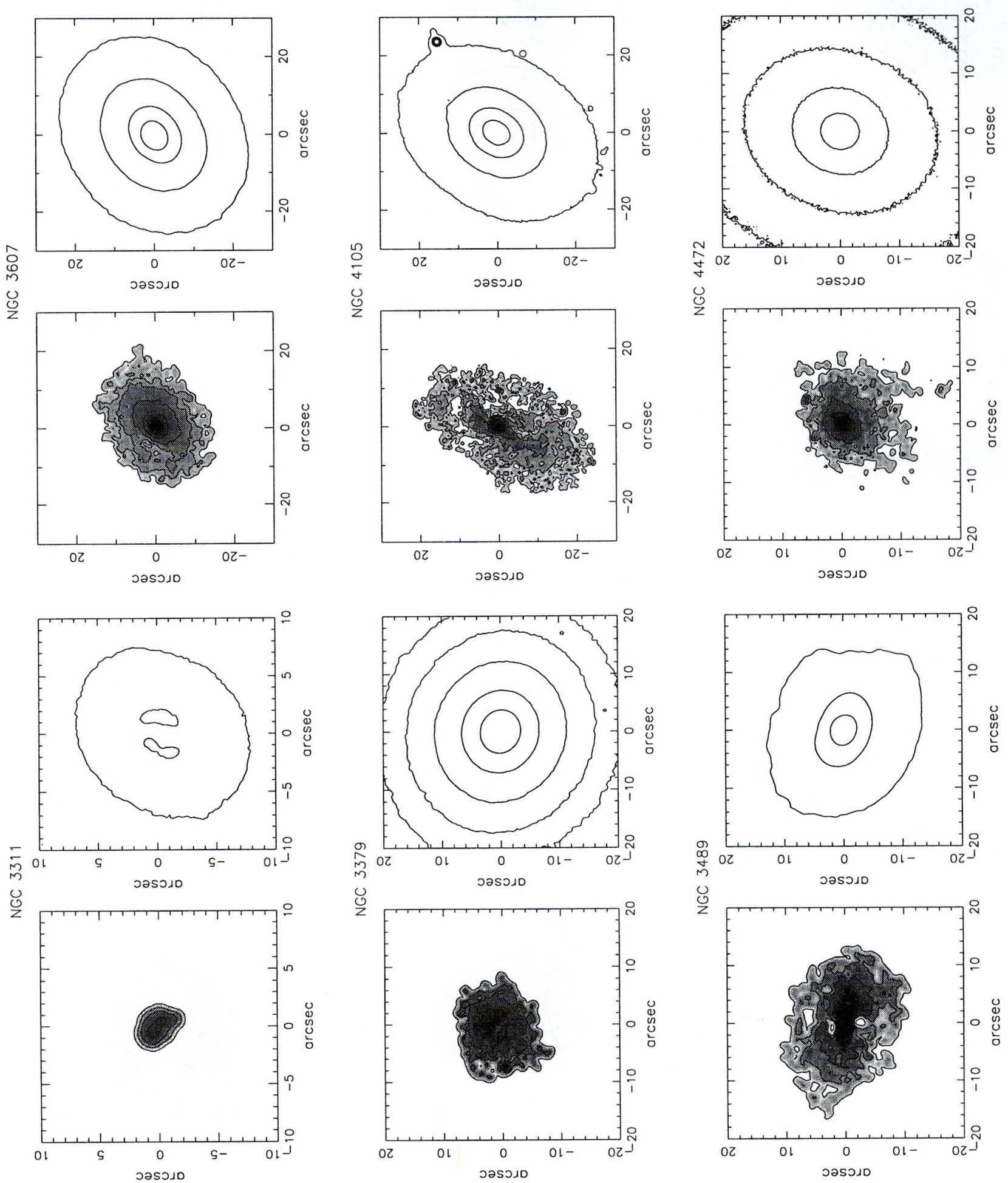

Fig. 9. NGC 3311. $\mathrm{H} \alpha+[\mathrm{NII}]: 0.6 ; R: 21.0 ; \mathrm{FWHM}=1$.' $^{\prime} ; \quad$ NGC 3379. H $\alpha+[\mathrm{NII}]: 0.6 ; R: 20.0 ; \mathrm{FWHM}=1{ }^{\prime \prime} 1 ; \quad$ NGC 3489. $\mathrm{H} \alpha+[\mathrm{NII}]: 1.7 ; R: 18.0 ; \mathrm{FWHM}=1 . \prime 6 ; \quad \mathrm{NGC} \mathrm{3607.} \mathrm{H} \alpha+[\mathrm{NII}]: 0.3 ; R: 20.0 ; \mathrm{FWHM}=2$. $^{\prime} 1 ; \quad \mathrm{NGC}$ 4105. H $\alpha+[\mathrm{NII}]$ :

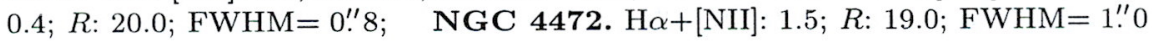



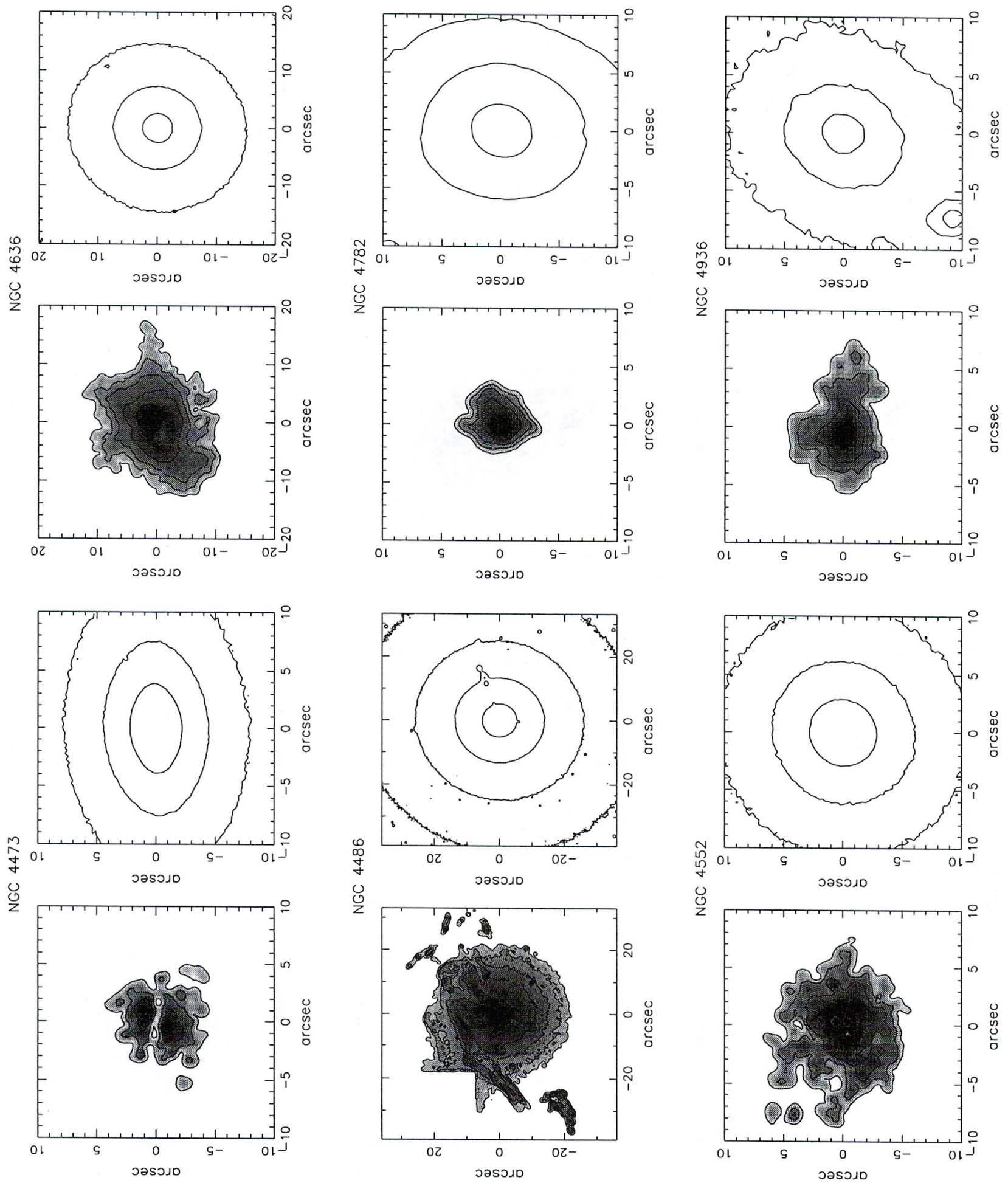

Fig. 9. NGC 4473. H $\alpha+[\mathrm{NII}]: 1.5 ; R: 18.0 ; \mathrm{FWHM}=1{ }^{\prime \prime} 0 ; \quad \mathrm{NGC}$ 4486. H $\alpha+[\mathrm{NII}]: 0.5 ; R: 22.0 ; \mathrm{FWHM}=0$." $8 ; \quad \mathrm{NGC}$

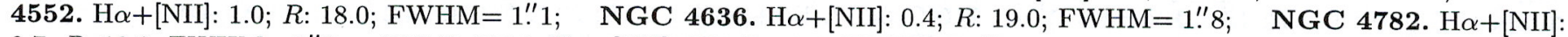
$0.7 ; R: 19.0 ; \mathrm{FWHM}=1 .{ }^{\prime \prime} 2 ; \quad$ NGC 4936. H $\alpha+[\mathrm{NII}]: 2.1 ; R: 19.0 ; \mathrm{FWHM}=1 .{ }^{\prime \prime} 0$ 

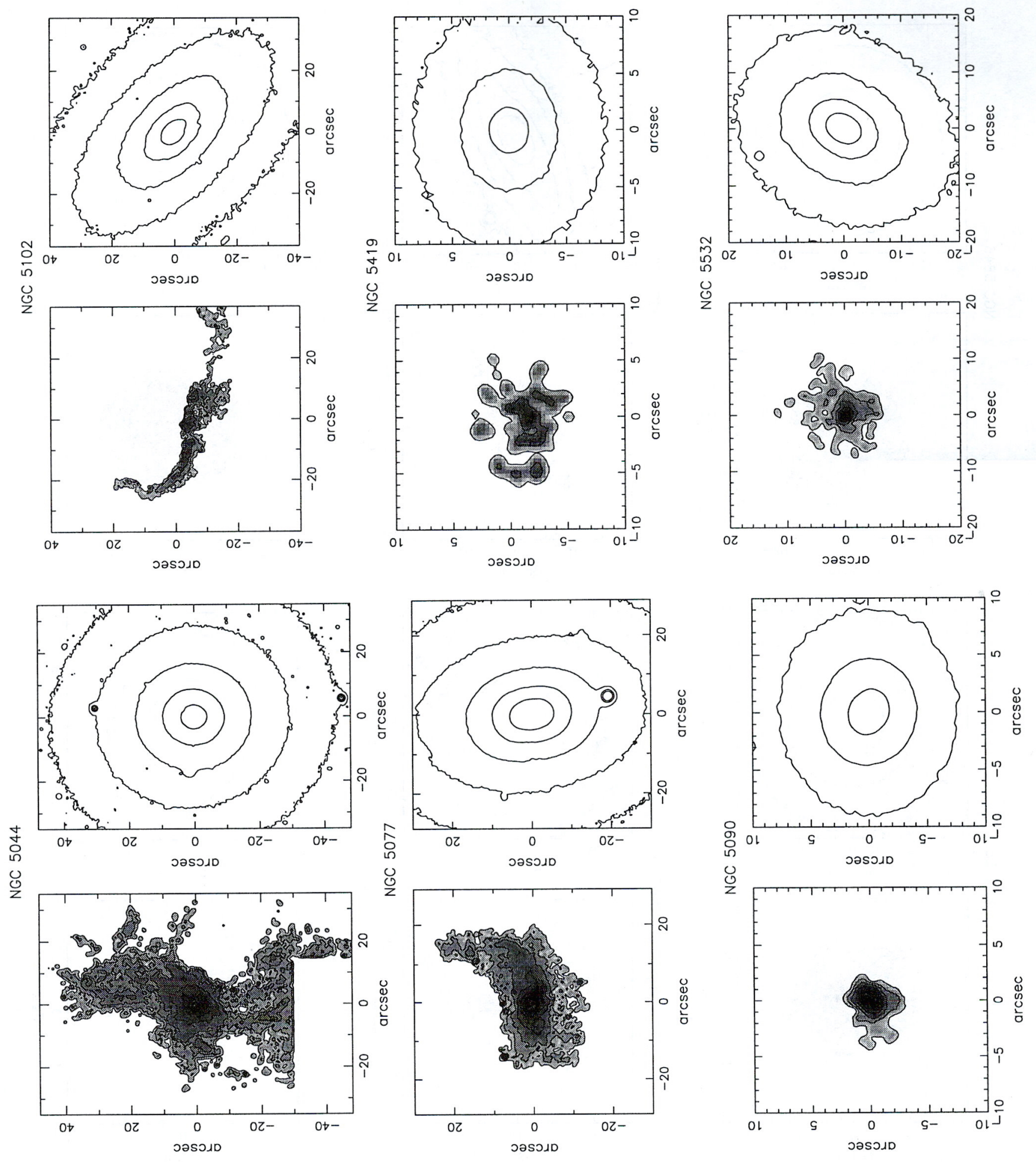

Fig. 9. NGC 5044. H $\alpha+[\mathrm{NII}]: 0.1 ; R: 22.0 ; \mathrm{FWHM}=1$. ." 0 . The removal of a ghost image mutilated the H $\alpha+[\mathrm{NII}]$ emission map in the lower part of the plot. NGC 5077. H $\alpha+[\mathrm{NII}]: 0.25 ; R: 22.0 ; \mathrm{FWHM}=1$." $^{\prime} ; \quad \mathrm{NGC} \mathbf{5 0 9 0}$. H $\alpha+[\mathrm{NII}]: 1.1 ; R: 19.0$; FWHM $=0 . " 9 ; \quad$ NGC 5102. H $\alpha+[\mathrm{NII}]: 1.2 ; R: 21.0 ; \mathrm{FWHM}=0 . ! 9 ; \quad$ NGC 5419. H $\alpha+[\mathrm{NII}]: 4.0 ; R: 19.0 ; \mathrm{FWHM}=1 . " 3 ;$

NGC 5532. $\mathrm{H} \alpha+[\mathrm{NII}]: 0.9 ; R: 21.0 ; \mathrm{FWHM}=1.2$ 

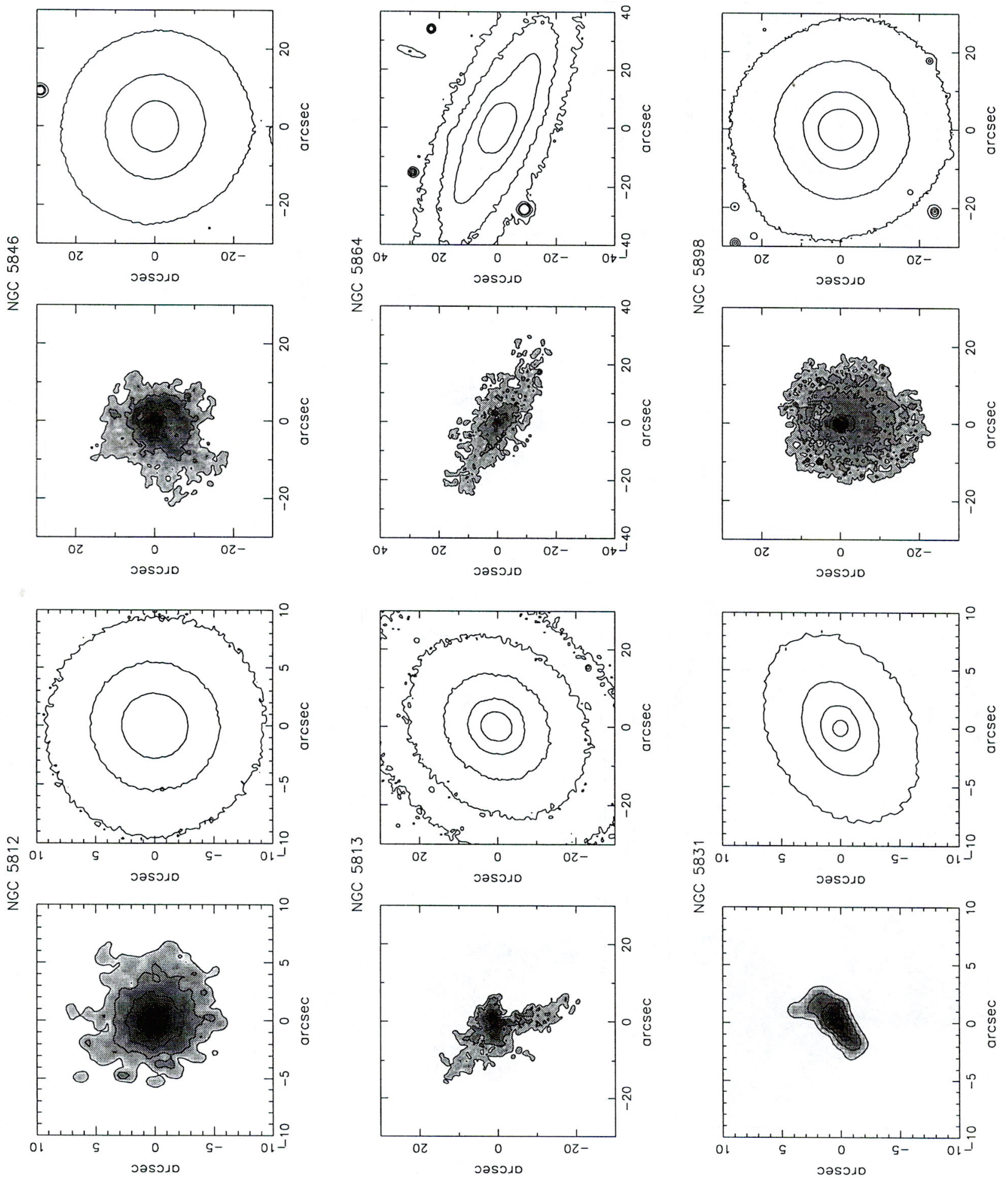

Fig. 9. NGC 5812. $\mathrm{H} \alpha+[\mathrm{NII}]: 1.2 ; R: 18.0 ; \mathrm{FWHM}=1 .{ }^{\prime \prime} 1 ; \quad$ NGC 5813. $\mathrm{H} \alpha+[\mathrm{NII}]: 1.2 ; R: 22.0 ;$ FWHM $=1 .{ }^{\prime \prime} 0 ; \quad \mathrm{NGC}$ 5831. $\mathrm{H} \alpha+[\mathrm{NII}]: 6.6 ; R: 19.0 ; \mathrm{FWHM}=1$. . $2 ; \quad$ NGC 5846. H $\alpha+[\mathrm{NII}]: 1.5 ; R: 21.0 ; \mathrm{FWHM}=1$. " 1 ; NGC 5864. H $\alpha+[\mathrm{NII}]$ : 1.0; $R: 22.0 ; \mathrm{FWHM}=1 .{ }^{\prime \prime} 5 ; \quad$ NGC 5898. $\mathrm{H} \alpha+[\mathrm{NII}]: 0.3 ; R: 21.0 ; \mathrm{FWHM}=1 .{ }^{\prime \prime} 0$ 


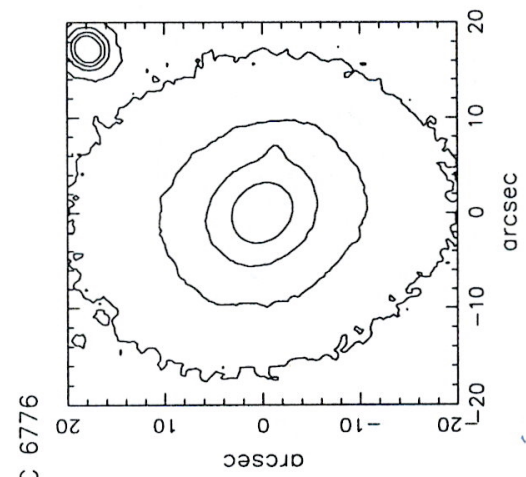

¿
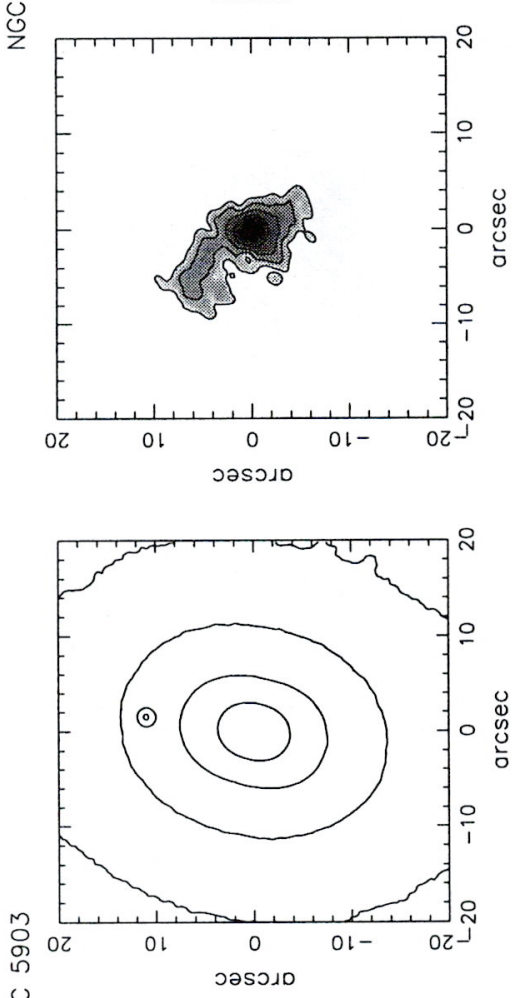

O

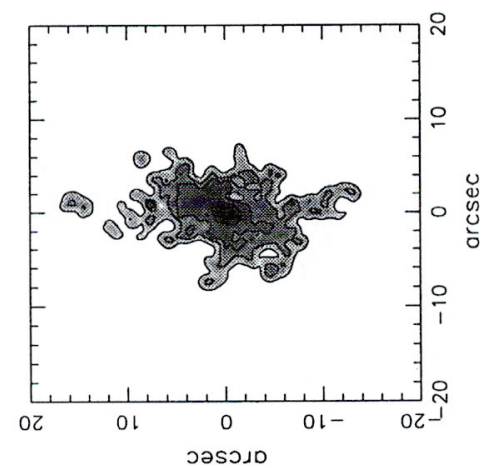

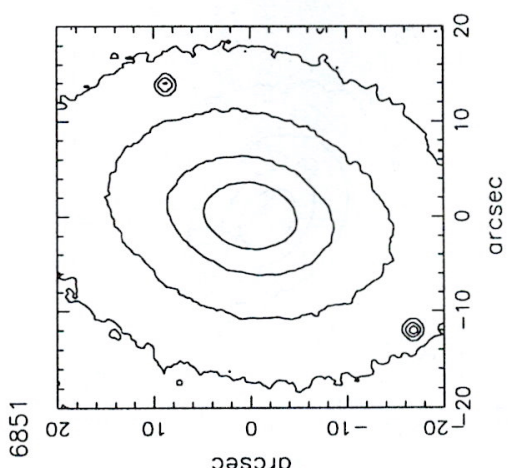

U
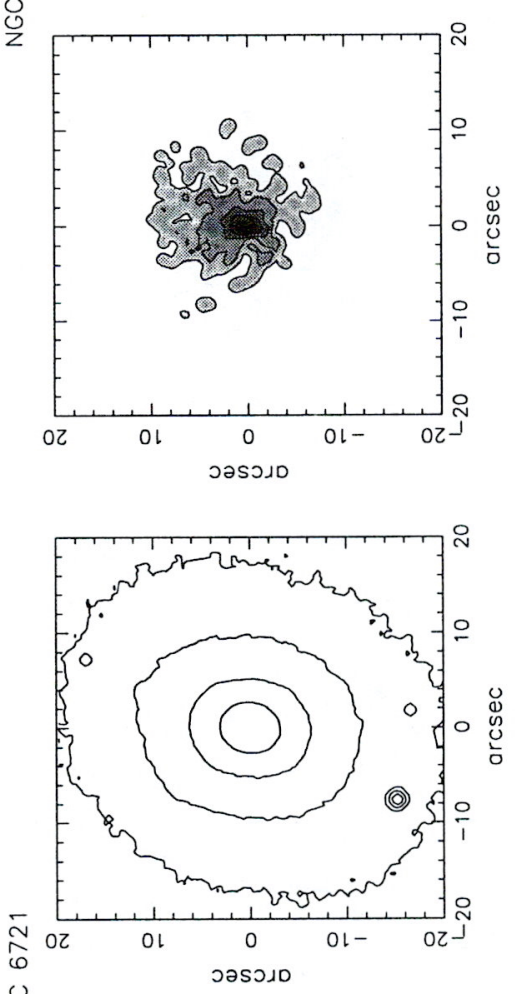

仓

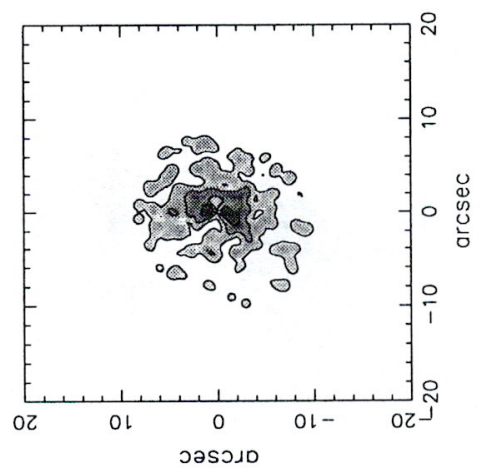

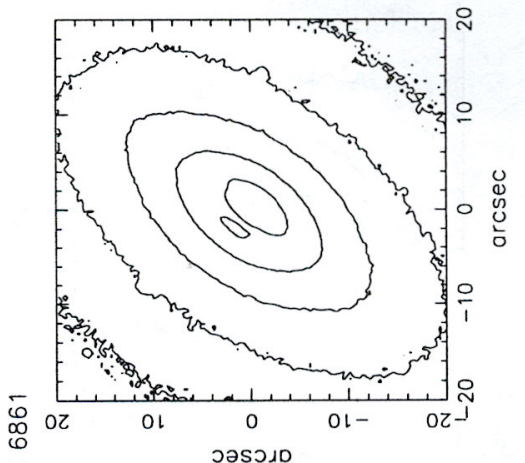

u
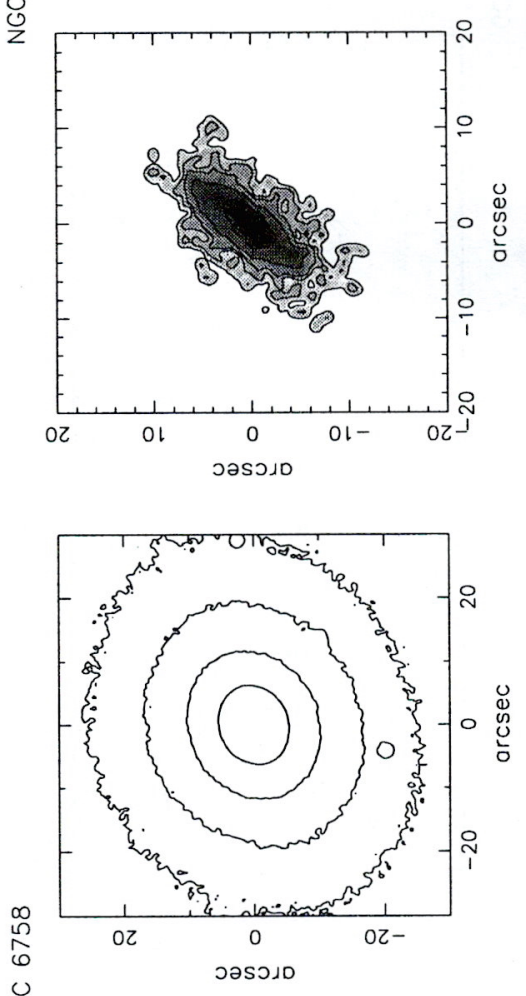

U

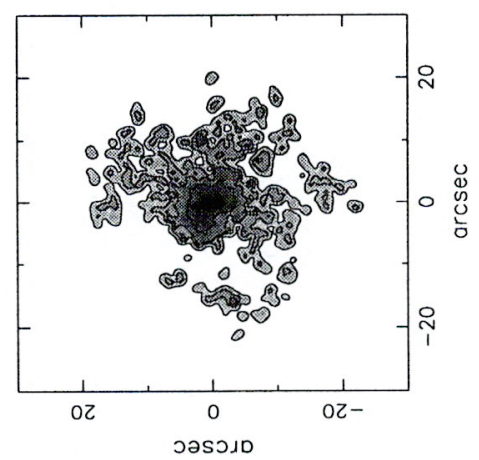

Fig. 9. NGC 5903. $\mathrm{H} \alpha+[\mathrm{NII}]: 0.5 ; R: 20.0 ; \mathrm{FWHM}=1 .{ }^{\prime \prime} 2 ; \quad$ NGC 6721. $\mathrm{H} \alpha+[\mathrm{NII}]: 3.4 ; R: 21.0 ; \mathrm{FWHM}=1{ }^{\prime \prime} 0 ; \quad \mathrm{NGC}$ 6758. $\mathrm{H} \alpha+[\mathrm{NII}]: 0.2 ; R: 22.0 ; \mathrm{FWHM}=1{ }^{\prime \prime} 6 ; \quad \mathrm{NGC} \mathrm{6776.} \mathrm{H} \alpha+[\mathrm{NII}]: 1.1 ; R: 21.0 ; \mathrm{FWHM}=1{ }^{\prime \prime} 3 ; \quad \mathrm{NGC} \mathrm{6851.} \mathrm{H} \alpha+[\mathrm{NII}]:$ $0.9 ; R: 21.0 ; \mathrm{FWHM}=0 .{ }^{\prime \prime} 9 ; \quad$ NGC 6861. $\mathrm{H} \alpha+[\mathrm{NII}]: 0.4 ; R: 21.0 ; \mathrm{FWHM}=1 .{ }^{\prime \prime} 0$ 

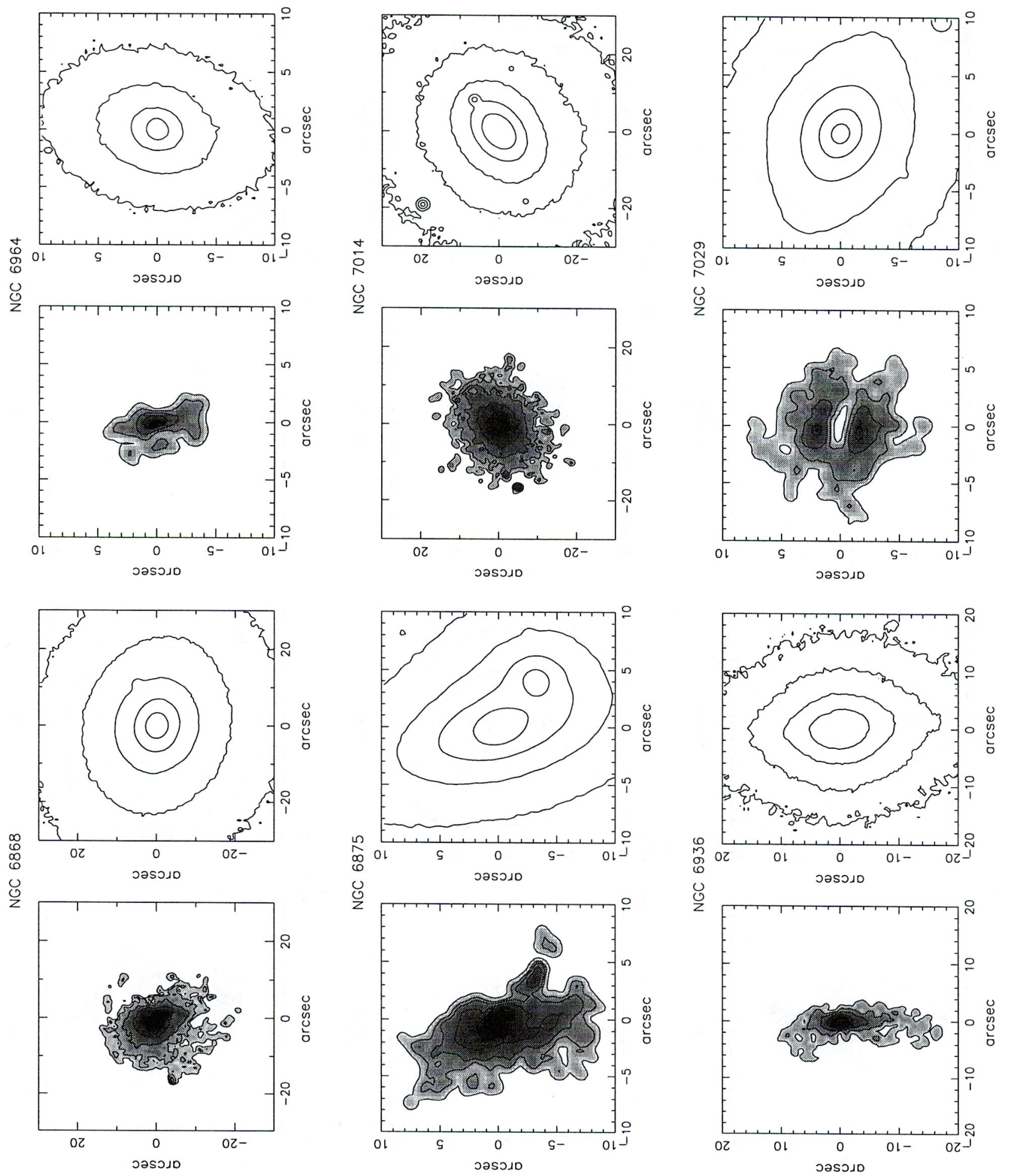

Fig. 9. NGC 6868. H $\alpha+[\mathrm{NII}]: 0.5 ; R: 21.0 ; \mathrm{FWHM}=1 .^{\prime \prime} 5 ; \quad \mathrm{NGC}$ 6875. H $\alpha+[\mathrm{NII}]: 0.2 ; R: 20.0 ; \mathrm{FWHM}=0$. $^{\prime} 9 ; \quad \mathrm{NGC}$ 6936. $\mathrm{H} \alpha+[\mathrm{NII}]: 1.0 ; R: 22.0 ; \mathrm{FWHM}=1 .{ }^{\prime \prime} 0 ; \quad$ NGC 6964. H $\alpha+[\mathrm{NII}]: 1.7 ; R: 20.0 ; \mathrm{FWHM}=1 .{ }^{\prime \prime} 0 ; \quad \mathrm{NGC} \mathbf{7 0 1 4}$. H $\alpha+[\mathrm{NII}]:$ $0.2 ; R: 23.0 ; \mathrm{FWHM}=1$. ." 4 ; NGC 7029. $\mathrm{H} \alpha+[\mathrm{NII}]: 0.5 ; R: 20.0 ; \mathrm{FWHM}=1{ }^{\prime \prime} 4$ 


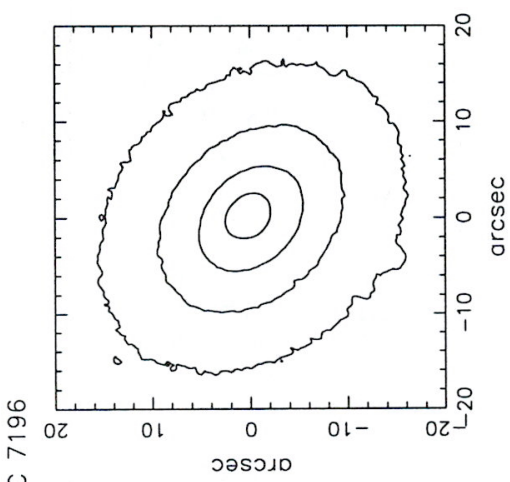

O
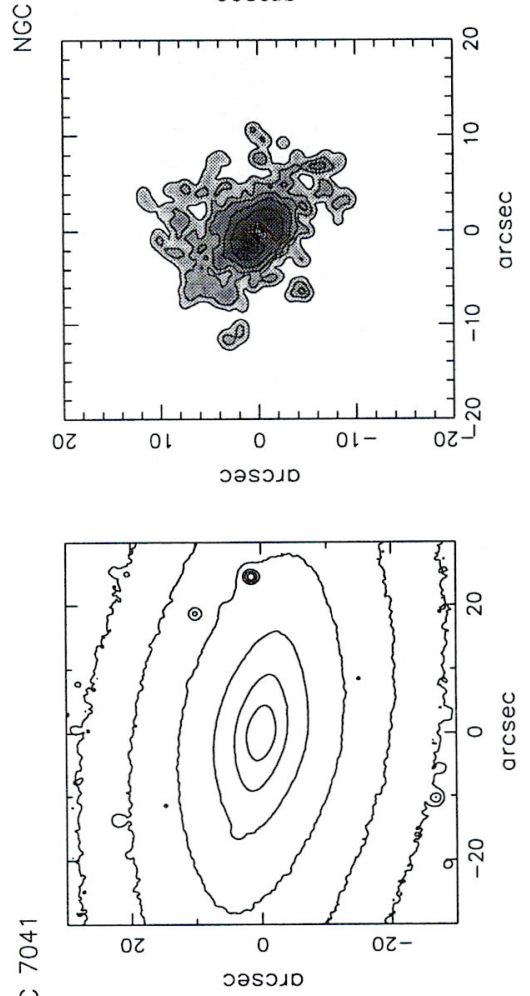

仓

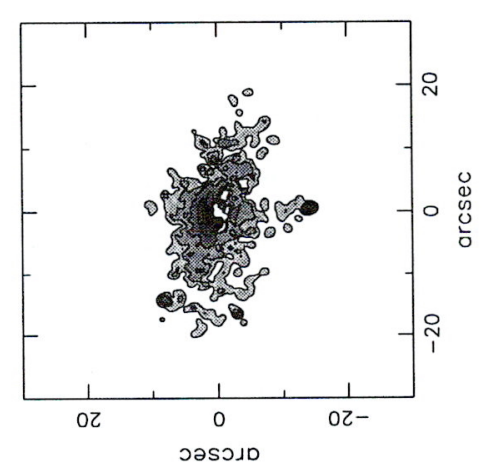

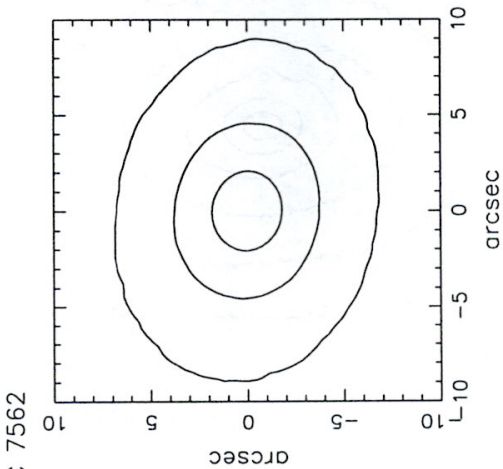

O
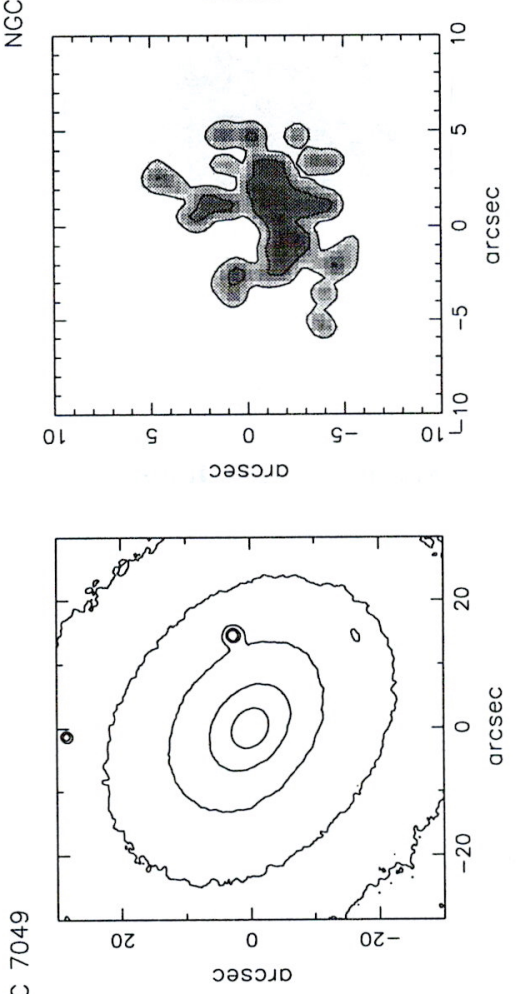

O

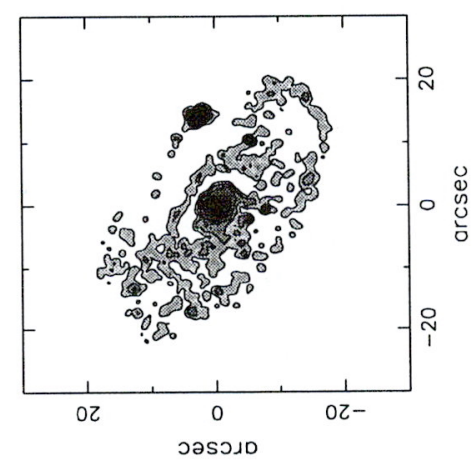

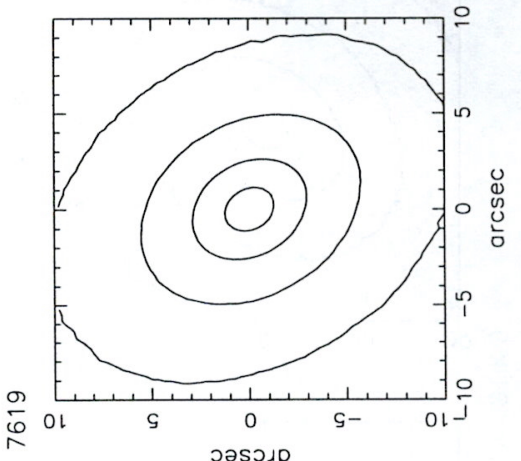

芩
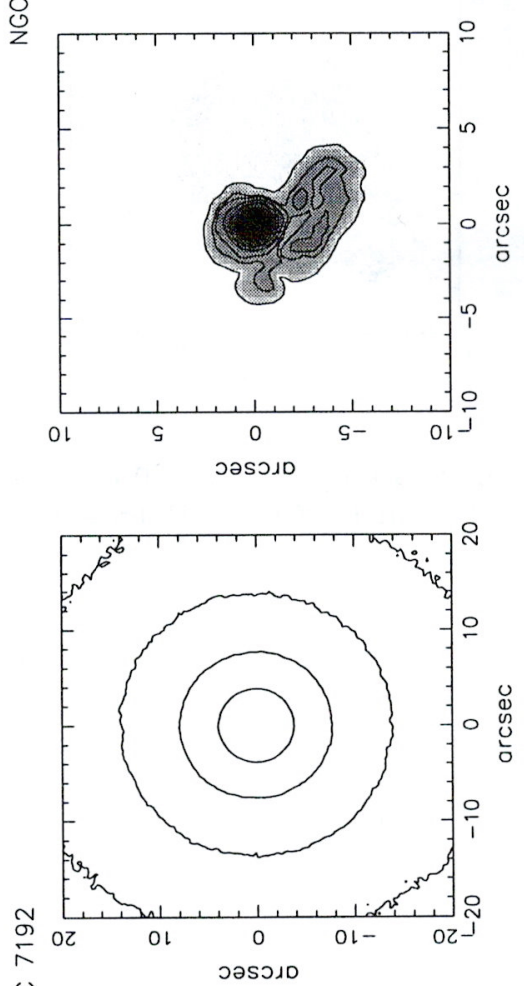

U

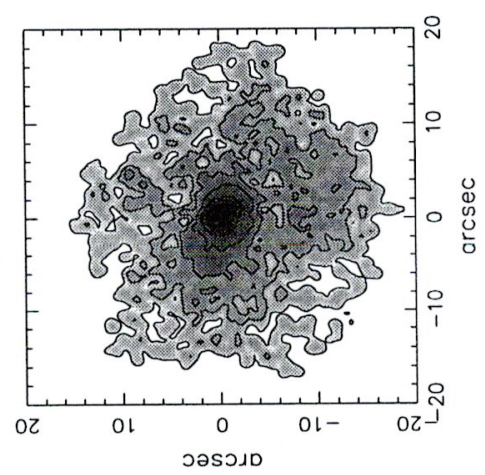

Fig. 9. NGC 7041. $\mathrm{H} \alpha+[\mathrm{NII}]: 0.3 ; R: 22.0 ; \mathrm{FWHM}=1$. " $1 ; \quad$ NGC 7049. $\mathrm{H} \alpha+[\mathrm{NII}]: 0.8 ; R: 21.0 ;$ FWHM=1."0; $\quad$ NGC 7192. $\mathrm{H} \alpha+[\mathrm{NII}]: 0.2 ; R: 21.0 ; \mathrm{FWHM}=1$. ." $8 ; \quad$ NGC 7196. $\mathrm{H} \alpha+[\mathrm{NII}]: 0.15 ; R: 21.0 ; \mathrm{FWHM}=1 . " 2 ; \quad$ NGC 7562. H $\alpha+[\mathrm{NII}]$ : 1.3; $R: 19.0 ; \mathrm{FWHM}=1 . " 4 ; \quad \mathrm{NGC} \mathrm{7619.} \mathrm{H} \alpha+[\mathrm{NII}]: 0.5 ; R: 19.0 ; \mathrm{FWHM}=1 . " 1$ 

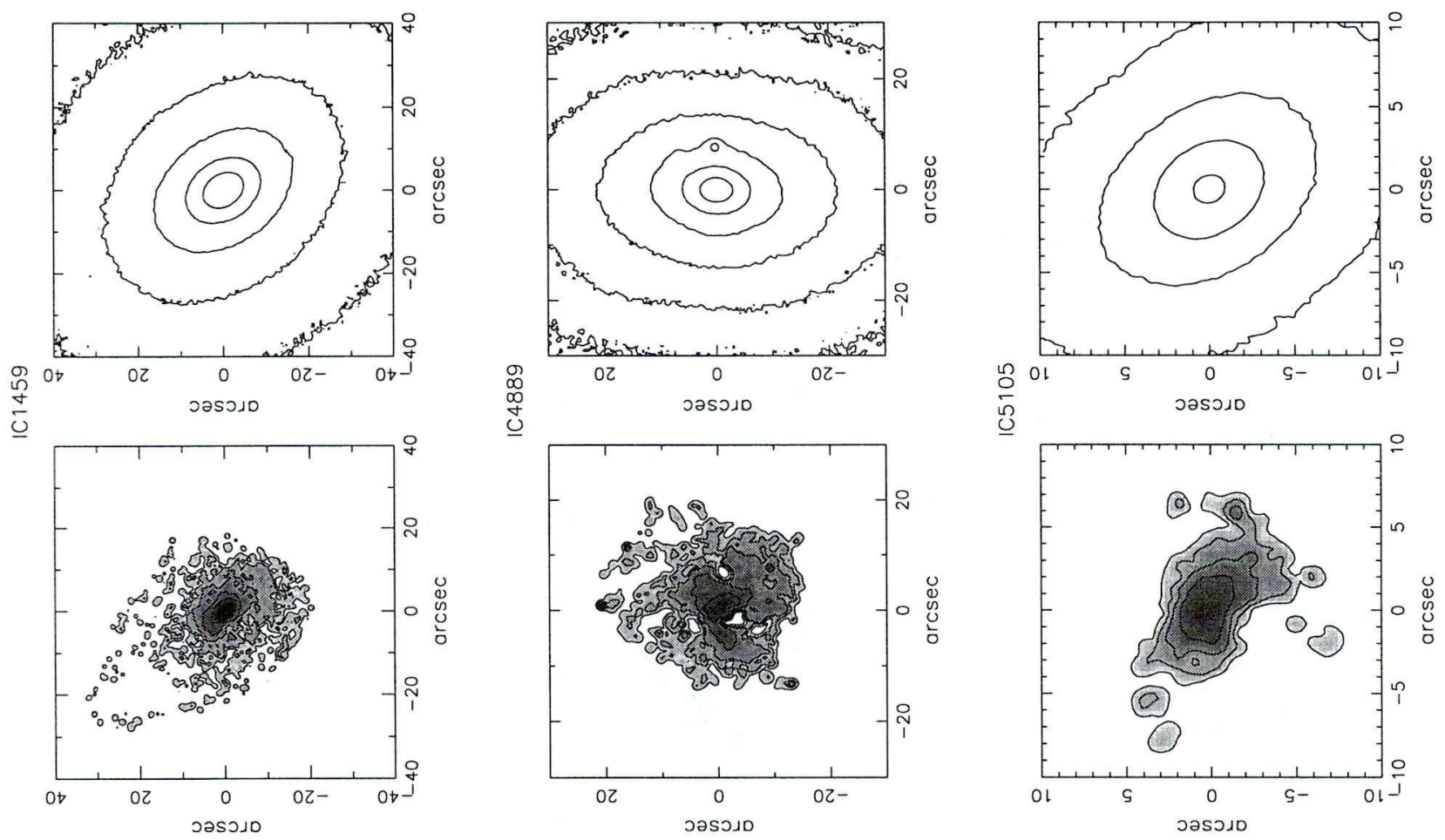

Fig. 9. IC 1459. H $\alpha+[\mathrm{NII}]: 0.7 ; R: 21.0 ; \mathrm{FWHM}=0 !^{\prime \prime} 9 ; \quad$ IC $4889 . \mathrm{H} \alpha+[\mathrm{NII}]: 0.15 ; R: 22.0 ; \mathrm{FWHM}=1{ }^{\prime \prime} 7 ; \quad$ IC $\mathbf{5 1 0 5}$. $\mathrm{H} \alpha+[\mathrm{NII}]: 0.4 ; R: 20.0 ; \mathrm{FWHM}=1 . " 8$ 